\title{
Trellis- and Network-Coded Modulation for Decode-and-Forward Two-Way Relaying Over Time-Varying Channels
}

\author{
Yanping Yang, Student Member, IEEE, Wei Chen, Senior Member, IEEE, Ou Li, Ke Ke, \\ and Lajos Hanzo, Fellow, IEEE
}

\begin{abstract}
We present a bandwidth-efficient joint channel coding-modulation scheme conceived for the broadcast channel of decode-and-forward (DF) two-way relaying (TWR), where trellis-coded modulation (TCM) is intrinsically amalgamated with network-coded modulation (NCM) for achieving both a channel coding gain and a high throughput. We conceive a lowcomplexity receiver algorithm for our joint TC-NCM scheme, which applies decoding and demodulation simultaneously, without the need to first demodulate the signal before decoding, as in the traditional solutions. As a further contribution, the TC-NCM scheme is intrinsically amalgamated with adaptive transceiver techniques. We then further investigate the performance of our near-instantaneously adaptive discrete-rate TC-NC-quadratureamplitude modulation/phase-shift keying (QAM-PSK) scheme. Both simulation results and numerical analysis are presented, which are compared with the performance of traditional NCM schemes. The results show that our scheme not only increases the achievable transmission rate but improves the reliability as well, yet it is of modest complexity.
\end{abstract}

Index Terms - Adaptive modulation, fading channels, networkcoded modulation (NCM), trellis-coded modulation (TCM), two-way relaying (TWR).

$\mathbf{W}$ ITHIN just a few decades, wireless communications have undergone a rapid growth from their initial conception to worldwide penetration, which has changed our daily lives as well as the way we think. Mobile communication has become the most important linkage between individuals and information networks. The increasing density of mobile users has

Manuscript received August 8, 2015; revised March 27, 2016; accepted September 30, 2016. Date of publication; date of current version. This work was supported in part by the the National Natural Science Foundation of China under Grant 61671269, Grant 61201380, Grant 61601516, Grant 61322111, and Grant 61321061; in part by the National High Technology Research and Development Program of China (863 Program) under Grant 2012AA121606; in part by the National Basic Research Program of China (973 Program) under Grant 2013CB336600 and Grant 2012CB316000; in part by the Engineering and Physical Sciences Research Council under Project EP/Noo4558/1 and Project EP/L018659/1; in part by the Tsinghua National Lab on Information Science and Technology; in part by the European Research Council's Advanced Fellow Grant through the Beam-Me-Up project; and in part by the Royal Society Wolfson Research Merit Award. The review of this paper was coordinated by Prof. J.-Y. Chouinard.

Y. Yang, W. Chen, O. Li, and K. Ke are with the Department of Electronic Engineering, Tsinghua University, Beijing 100084, China (e-mail: y.p.yang1986@ gmail.com; wchen@mail.tsinghua.edu.cn; zzliou@126.com; kek2015@126. com).

L. Hanzo is with the Department of Electronics and Computer Science, University of Southampton, Southampton SO17 1BJ, U.K. (e-mail: 1h@ecs. soton.ac.uk).

Digital Object Identifier 10.1109/TVT.2016.2615656 fueled an escalating demand for higher capacity and reliability of the network. Relaying combined with other powerful physical layer transmission techniques are capable of significantly improving the achievable spectrum efficiency and/or expanding the range of high-throughput cellular coverage. As an attractive solution, network coding (NC) [1], [2], which was originally proposed for wired networks, is capable of significantly improving a wireless relaying network's throughput and robustness. Since 2000, diverse NC techniques have been conceived for multiuser communication relying on relaying [3]-[13]. To the best of our knowledge, the treatise of Wu et al. [3] was the first NC-contribution on the practical subject of simultaneous two-way information exchange between two nodes. NC methods conceived for multiuser communications were investigated in [4]-[6], where the relay node (RN) of two-way relaying (TWR) that performs an XOR operation on the decoded bit stream was presented in [4], with the upper and lower frame error ratio performance bounds of cooperative multiuser systems using NC derived in [5], while noncoherent near-capacity $\mathrm{NC}$ schemes relying on extrinsic information transfer charts designed in [6]. NC techniques for TWR channel were developed in [7]-[13]. Thereinto, Popovski and Yomo [7] explored several methods invoking physical-layer NC for the TWR channel, Xie [8] and Wu [9] investigated the downlink capacity of asymmetric $^{1}$ decode-and-forward TWR (DF-TWR). More explicitly, Larsson [10] provided a low-complexity XOR-based NC en-/decoding method, while Manssour et al. conceived a generalized symbol-level multiplicative NC scheme in [11], where $\mathrm{NC}$-quadratic-amplitude modulation (QAM) was considered in detail. Furthermore, they proposed an $\mathrm{NC}$-aware link adaptation scheme for the wireless broadcast channel (BC) and combined it with XOR-based NC and generalized multiplicative NC in [12], which is capable of achieving a significantly improved throughput. Further research on asymmetric DF-TWR with NC modulation (NCM) was conducted by Chen et al. [13], where set-partitioning-based NCM and a NC-oriented maximum ratio combining (NC-MRC) scheme was conceived for the sake of maximizing the throughput, while achieving a beneficial diversity gain. NCM proposed in [11]-[13] have laid the foundations of asymmetric transmission research for TWR. Additionally, in [12] and [13], Manssour et al. and Chen et al. conceived adaptive NCM based on variable-rate transmissions, which motivates us

\footnotetext{
${ }^{1}$ The asymmetry here implies that the two traffic flows may have different symbol rates.
} 
to further investigate the family of adaptive NCM techniques designed for DF-TWR over time-varying channels.

Near-instantaneously adaptive modulation is capable of realizing reliable communications over hostile fading channels More explicitly, provided that the channel's complex envelope is known at the transmitter, an increased throughput can be achieved by adapting the transmit power, data rate, and coding scheme according to the near-instantaneous fading level [14]. A substantial amount of in-depth research has been dedicated to this topic [15]-[20]. Torrance and Hanzo [15] designed a set of optimum mode-switching levels, which was found for a generic constant-power adaptive-modulation scheme based on a specific target bit-error-rate (BER) by maximizing the achievable bits-per-symbol throughput. Goldsmith and Chua [16] proposed variable-rate variable-power transmission using uncoded $M$-ary QAM (MQAM), while coded adaptive MQAM was investigated in [17]. Channel coding is of crucial importance in wireless research [18]-[20]. Thereinto, Yee et al. characterized Turbo-coded adaptive MQAM in [18]. Both space-time trellis and space-time block coding were investigated in [19]. Hanzo et al. [20] conducted in-depth research on adaptive-coded modulation conceived for time-division multiple access (TDMA), code division multiple access, and OFDM systems. In a nutshell, during the 2000s, these solutions have found their way into literally all wireless standards. Furthermore, they provided two directions for our study of DF-TWR. One direction is the joint power and rate adaptation, which was proposed in [13] for NCM to improve the bandwidth efficiency of relaying network. The other direction is the joint design of channel coding with $\mathrm{NCM}$, which holds the potential of significantly improving the network's robustness.

As for the first direction, Chen et al. [13] have explored constant-power, variable-rate adaptive NCM, where only the rates are time-variant, subject to the channel conditions. A combination of the techniques advocated in [13] and [16] was invoked for DF-TWR's downlink in [21], where joint variablepower and variable-rate schemes were investigated in order to improve the throughput of networks. However, most of these adaptive solutions were designed without considering spectrally efficient coding, with the exception of [17] and [18].

We continue by considering the second direction for TWR. Both channel coding and modulation techniques have to be designed for maintaining a certain target-integrity, but these two techniques are often designed separately. Hence, the joint design of channel coding, adaptive modulation, and $\mathrm{NC}$ for DF-TWR is still in its infancy and currently there is a paucity of contributions on related research. Inspired both by the trellis-coded MQAM philosophy of [17] and the adaptive turbo-trellis-coded modulation-aided asymmetric distribution source coding of [22], we conceived a novel coding scheme termed as trellis- and network-coded modulation (TCNCM). Explicitly, we intrinsically amalgamate both adaptive NCM [21] and TCM [23], [24] for the downlink of DF-TWR, so that both the information transmission rate and the reliability are improved. Since the achievable channel coding gain is essentially independent of the selection of modulation [17], we can adjust the transmit power, the two links' coding design, as well as the pair of transmit rates at the RN to maximize the average data rate without affecting the BER performance and the coding gain.

Against this background [13], [16], [17] and [21], we would like to summarize our main contributions as follows:

1) Peer-to-peer versus two-way relay channel: We extend the single-link peer-to-peer regime of [16], [17] to the DF-TWR scenario, in which the transmit power at the RN has to simultaneously adapt to a pair of potentially different channel conditions, rather than to a single link.

2) An intrinsic amalgam of TCM and NCM: We adapt the standalone TCM [17] and the standalone NCM concepts [13], [21] by intrinsically amalgamating them into a new, inseparable, and more powerful scheme without requiring any bandwidth expansion. This powerful combination of NCM with bandwidth-efficient TCM leads to a joint encoder structure associated with the pair of bidirectional links of two-way relaying.

3) Joint design of our NC-aided trellis-coding algorithm: By exploiting the innate structure of TCM, we develop a joint TC-NCM scheme. Additionally, in contrast to traditional solutions [17], joint decoding and demodulation is conceived, which operates without the need to first demodulate the signal before decoding.

4) Limitations imposed on the joint encoder: A specific constraint of our near-instantaneously adaptive DF-TWR technique is that a signal-to-noise ratio (SNR)-loss is imposed by NC-QAM, which is analyzed.

The remainder of this paper is organized as follows. We commence by describing both the system and the channel model of DF-TWR in Section II. We then conceive our generic structure of TC-NCM in Section III, where the motivation, the transmitter design, as well as the data flow are detailed. Based on the proposed structure, we develop the transmission mechanism of TC-NCM in Section IV, which is followed by the performance analysis of the proposed adaptive TC-NC-QAM/phaseshift keying (PSK) scheme in Section V. Finally, we present our simulation and numerical results, characterizing the new scheme in Section VI, with our concluding remarks provided in Section VII.

\section{System AND Channel Models}

Consider a typical asymmetric DF-TWR scenario. The timedivision multiplexing/TDMA two-way relay communication system is shown in Fig. 1, where the users are multiplexed to transmit in different time slots. The two destination nodes (DN1 and DN2) wish to exchange information between each other via the RN, where it is assumed that the channel is bidirectional and half-duplex so that transmission and reception at each node must take place in different time slots. The typical DF-TWR transmission can be divided into two distinct stages: 1) the multiple access (MA) stage when Source Node 1 and Source Node 2 (SN1 and SN2) separately send their data to the RN and 2) the BC stage, when the RN broadcasts the processed signal to both DN1 and DN2. In particular, each DN has a priori knowledge of its own message intended for the other. Throughout this paper, 


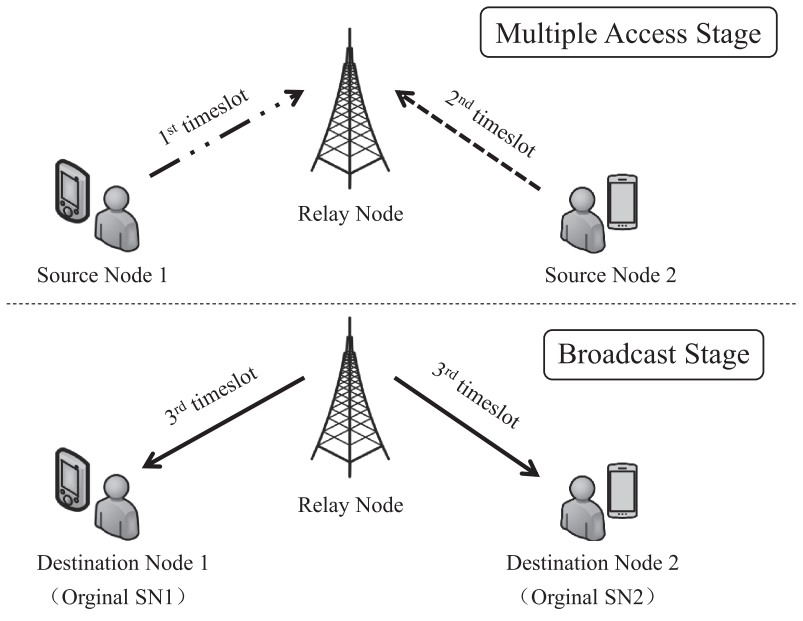

Fig. 1. Three time-slot DF-TWR.

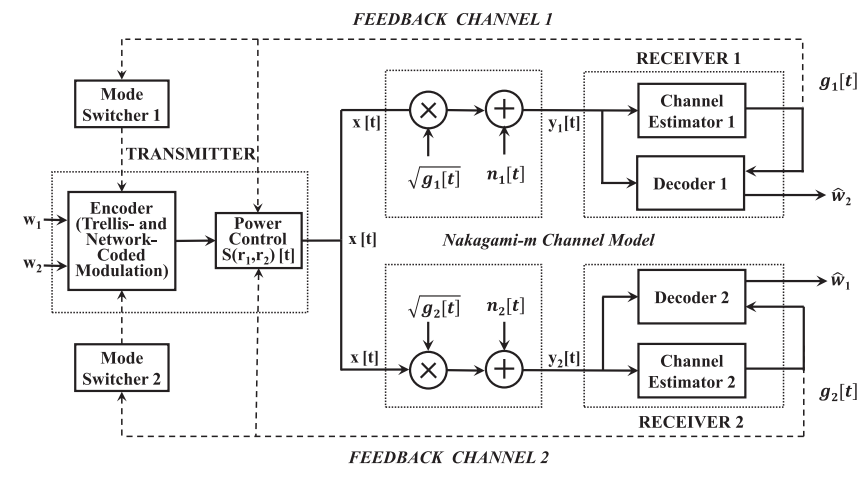

Fig. 2. System and channel models (broadcast stage).

we only focus our attention on the BC stage, where it is assumed that the RN has already successfully received the signals from the two source nodes during the MA stage. ${ }^{2}$

Building on the BC stage of Fig. 1, our overall TC-NCM design is shown in Fig. 2, where both the general TC-NCM system and the channel model are presented. The complete data flow contains input signal $W_{i}, i=1,2$, NC symbols $x[t]$, received signals $y_{i}[t]$, as well as output signals $\hat{W}_{i}[t]$, which will be addressed in detail in Section III-C. Here, we first present our channel model based on [16]. It is assumed that the system uses ideal Nyquist sampling, where $B=1 / T_{s}$ denotes the bandwidth and $T_{s}$ is the symbol duration. Similar to the channel model of [16], the channel has a real-valued stationary and ergodic multiplicative gain $g_{i}[t]$ and imposes complex-valued additive white Gaussian noise (AWGN) $n_{i}[t]$. For convenience, it defines the random variables $\gamma_{i}[t]=\bar{S} g_{i}[t] /\left(N_{0_{i}} B\right)$ associated with the means $\overline{\gamma_{i}}=\bar{S} /\left(N_{0_{i}} B\right)$ and distribution of $p\left(r_{i}[t]\right)$, which represent the channel SNR [16], while $\bar{S}$ denotes the average transmit power. Furthermore, $N_{0_{i}}$ denotes the noise power spectral density. When the context is unambiguous, we will omit the time reference $t$ related to $g_{i}, \gamma_{i}$, and $\overline{\gamma_{i}}$.

Since the channel is time-variant, we adopt the general Nakagami- $m$ model for describing $\gamma_{i}$ statistically, with the

${ }^{2}$ During the MA stage, SN1 and SN2 transmit in two different time-slots to avoid their mutual interference [13]. fading distribution $p\left(\gamma_{i}\right)$ given by (see [25] and [26, Ch. 3.2])

$$
p\left(\gamma_{i}\right)=\frac{m^{m} \gamma_{i}^{m-1}}{{\overline{\gamma_{i}}}^{m} \Gamma(m)} \exp \left(-\frac{m \gamma_{i}}{\overline{\gamma_{i}}}\right), i=1,2
$$

where $\gamma_{i}$ represents the instantaneous SNR, $\bar{\gamma}_{i}$ denotes the aver- 213 age $\mathrm{SNR}, \Gamma(m):=\int_{0}^{\infty} t^{m-1} e^{-t} \mathrm{dt}$ is the Gamma function, and 214 $m$ is the Nakagami fading parameter. We choose the Nakagami- 215 $m$ distribution, because it is mathematically convenient and can 216 be applied for modeling a large class of fading channels, without 217 having to derive separate equations for the AWGN, Rayleigh and 218 Ricean probability distribution function. Explicitly, it includes 219 the Rayleigh channel as a special case, when $m=1$. Addition- 220 ally, a one-to-one mapping between the Ricean factor and the 221 Nakagami fading parameter $m$ allows also Ricean channels to 222 be closely approximated by Nakagami- $m$ channels.

Having outlined the transmission model, next we list all of our operating assumptions used throughout this paper.

A1) We consider slowly varying nondispersive fading channels. If the channel is changing faster than the rate at which it can be estimated and fed back to the transmitter, adaptive techniques will perform poorly. Therefore, it is assumed that the constellation size (transmit rate) must remain constant over hundreds of symbols. Since the constellation size is adapted to an estimate of the channel's fading level, dozens of symbol durations may be required to obtain a reliable estimate.

A2) Perfect channel state information is available both at the RN and DNs. It is assumed that the pair of feedback path does not introduce any errors, which can be approximately satisfied, provided that sufficiently powerful error correction and detection codes are used on the feedback path.

A3) It is assumed that the feedback path delays are $\tau_{i}=$ $0, i=1,2$. The effects of feedback path delays on adaptive modulation were analyzed in [16], where it was found that a feedback path delay of less than $0.001 / f_{D}$ only results in a modest performance degradation.

A4) For practical MQAM, it is required that the signal constellations are restricted to $M_{j}=2^{2 \tilde{k}}, j=1,2 ; \tilde{k}=$ $2,3, \ldots$, which implies that the coset codes employed have a zero constellation shaping gain. Additionally, the signal constellations of $M$-ary PSK (MPSK) are restricted to $M_{j}=2^{\tilde{k}}, j=1,2 ; \tilde{k}=2,3, \ldots$.

\section{COMbining TREllis-Coded Modulation With NETWORK-CODED MODULATION}

Based on the system and channel model of Fig. 2, we further 254 develop our joint TCM and NCM design in Figs. 3 and 4.

\section{A. Motivation of the Structure}

Let us recall the salient characteristics of TCM. It is well 257 known that Ungerboeck's scheme [24] combines coding and 258 modulation by expanding the Euclidean distance (ED) between 259 codewords and absorbs the parity bits without bandwidth ex- 260 pansion by doubling the number of constellation points due 261 to increasing the number of bits/symbol by one. This design 262 


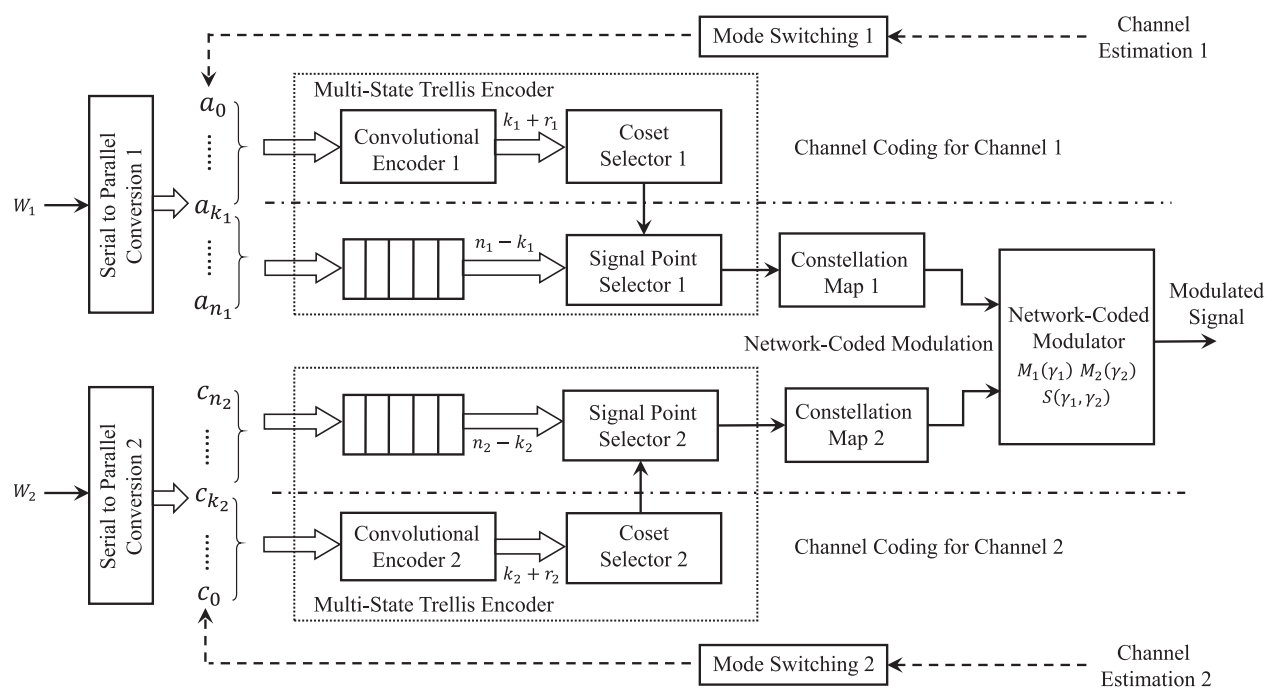

Fig. 3. Transmitter design of adaptive TC-NCM.

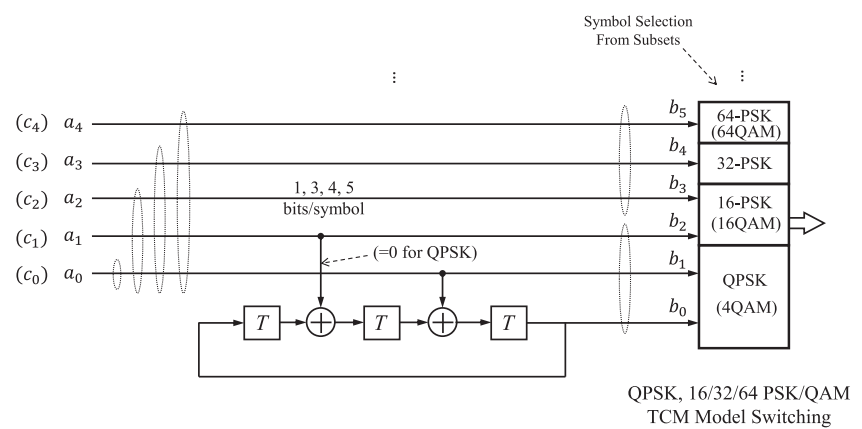

Fig. 4. Structure of the multirate trellis-based convolutional encoder (eightstate QPSK(QAM)/16PSK(16QAM)/32PSK/64PSK(64QAM)-TCM). jointly optimizes both channel coding and modulation, hence again, resulting in significant coding gains with no bandwidth expansion.

Based on the general type of coset coding advocated by Goldsmith [17] and [26, ch. 8], we develop our adaptive TC-NCM structure of Fig. 3, where the transmitter adapts the coding rate and modulation mode according to the channel estimates fed back via feedback channels, by adjusting $a_{0}, a_{1}, \ldots, a_{n_{1}}$ and $c_{0}, c_{1}, \ldots, c_{n_{2}}$. More explicitly, the channel coding of Fig. 4 is constituted by a convolutional encoder, with the modulation relies on symbol-based NCM. This intrinsically amalgamated structure enables us to achieve both a channel coding gain and all the NC benefits simultaneously.

\section{B. Transmitter Design}

Inspired by the general design of coset coding [26, Chapter 8.7] and by the system model of Fig. 2, we conceive the general architecture of our TC-NCM-aided transmitter design in Fig. 3. The function of each module is described as follows:

1) Convolutional Encoder operates on $k$ uncoded data bits to produce $k+r$ coded bits, which is the basic component of TCM. In our design, we use Ungerboeck's heuristics
[24] to design the trellis structure and the bit-to-symbol 284 assignment for four- and eight-state codes. 285

2) Coset Selector uses the coded bits to choose one of the 286 $2^{k+r}$ subsets from a partition of the $M$-ary constellation. 287

3) Signal Point Selector uses the uncoded bits to choose one 288 of the $2^{n-k}$ signal constellation points.

4) Constellation Map maps the selected point from $N-290$ dimensional space to a sequence of $N / 2$ points in a 291 two-dimensional space.

5) Network-Coded Modulator employs the NCM algorithm to generate the modulated symbols.

The multirate trellis encoder plays a central role in the transmitter design. Fig. 4 gives an example of the classic eight-state TCM multirate encoder, where the number of bits/symbol can be adapted in unison with the pair of near-instantaneous SNRs $\gamma_{1}$ and $\gamma_{2}$.

\section{Data Flow of TC-NCM}

In accordance with the above design, we will next detail the 301 data flow in our proposed TC-NCM scheme.

1) Adaptive Trellis Codes: The messages received at the RN 303 during the MA stage are denoted by $W_{1}$ and $W_{2}$, as shown in 304 Fig. 2, where the serial-to-parallel converter converts a number 305 of bits into symbols. Let us assume that based on the pair of 306 channel condition, we have already determined both the coding 307 rates and constellation sizes $\left(M_{1}\right.$ and $\left.M_{2}\right)$. The parallel signals 308 (take $a_{i}$ for example) $a_{0}, a_{1}, \ldots, a_{n_{1}}$ will be encoded by the 309 trellis encoder according to $M_{1}$. Then, a specific symbol will 310 be jointly generated by the coset selector and the bit-to-symbol 311 mapper. Here, adaptation signifies that the coding modes vary 312 with the instantaneous SNR. Additionally, we have to point out 313 a specific feature of our design, because for the pair of downlink, 314 we have to first determine the most appropriate transmit mode 315 of one of the links, while the mode of the other link will depend 316 on the above-mentioned mode already determined.

2) Generate an NCM Symbol: Having generated both the 318 downlink symbols by the pair of trellis encoders, let us now 319 
continue by describing our symbol-based NC-QAM/PSK method of [13]. As for the static asymmetric DF-TWR's downlink, the equivalent baseband signals received at the coherent receiver of DN1 and DN2 are represented by

$$
Y_{i}=h_{i} X+N_{i}, i=1,2
$$

where $Y_{i}$ denotes the received modulated signal, with $\left|h_{i}\right|^{2}=g_{i}$ denotes the channel gains, $X$ denotes the transmit symbol at the RN. For the discrete-time downlink channel with $t$ denoting the time instants, the variables in (2) can be represented by $y_{i}[t]$, $x[t], \sqrt{g_{i}[t]}$, and $n_{i}[t]$, as shown in Fig. 2. For convenience, the following discussions consider the static case for demonstration. Relying on the universal NCM method based on the classic setpartitioning philosophy of [13], the detailed generations of the transmit symbol $X$ for NC-QAM/PSK are described below.

The constellation sizes of the two downlink traffic flows are denoted by $M_{1}, M_{2}$, supposing $M_{2} \geq M_{1}, M_{2} / M_{1}=\mathbb{N}$. The messages $W_{1}, W_{2}$ are mapped to amplitude/phase points corresponding to $M_{1}$ and $M_{2}$ by the bit-to-symbol constellation mapper. Then the pair of amplitude/phase symbols are merged into a single signal $X$ using the modulo-two operation at the RN. We briefly elaborate on our NC-QAM/PSK scheme following these steps. The NC-QAM symbol is generated by obeying the following steps:

Step 1: Given an MQAM constellation size, determine $A_{i}$ from

$$
A_{i}=\left\{0, \frac{1}{\sqrt{M_{i}}}, \ldots, \frac{\sqrt{M_{i}}-1}{\sqrt{M_{i}}}\right\}, i=1,2 .
$$

Step 2: Formulate the constellation points from

$$
\chi_{i}=\left\{2 \sqrt{M_{i}}\left(a_{i}^{I}+j a_{i}^{Q}\right)-\left(\sqrt{M_{i}}-1\right)(1+j): a_{i}^{I}, a_{i}^{Q} \in A_{i}\right\}
$$

with $a_{i}^{I}$ and $a_{i}^{Q}$ denoting the amplitudes of the constellation points.

Step 3: Process the normalized amplitudes $\left(a_{1}^{I}, a_{1}^{Q}\right)$ and $\left(a_{2}^{I}, a_{2}^{Q}\right)$ from

$$
\left\{\begin{array}{l}
a^{I}=a_{1}^{I}+a_{2}^{I} \bmod 1 \\
a^{Q}=a_{1}^{Q}+a_{2}^{Q} \bmod 1 .
\end{array}\right.
$$

Step 4: Generate the NC-QAM symbols as ${ }^{3}$

$$
X=d\left[2 \sqrt{M_{2}}\left(a^{I}+j a^{Q}\right)-\left(\sqrt{M_{2}}-1\right)(1+j)\right] .
$$

The NC-PSK symbol is generated by the steps below:

Step 1: Given the MPSK constellation size, determine $\Theta_{i}$ as

$$
\Theta_{i}=\left\{0, \frac{2 \pi}{M_{i}}, \ldots, \frac{2\left(M_{i}-1\right) \pi}{M_{i}}\right\}, i=1,2 .
$$

Step 2: Identify a normalized MPSK constellation point by $\theta_{1}$ and $\theta_{2}$ as follows:

$$
\chi_{i}=\left\{\cos \theta_{i}+j \sin \theta_{i}: \theta_{i} \in \Theta_{i}\right\} .
$$

\footnotetext{
${ }^{3} d=\sqrt{\left(\left(3 E_{s}\right) / 2\left(M_{2}-1\right)-1\right)}, M_{2}>M_{1}$, with $d$ denoting half of the constellation-spacing in QAM, while $E_{s}$ denotes the symbol energy.
}

Step 3: Generate the symbol's phase $\theta$ according to

$$
\theta=\theta_{1}+\theta_{2} \bmod 2 \pi .
$$

Step 4: Generate the NC-PSK symbol as

$$
X=\sqrt{E_{s}}(\cos \theta+j \sin \theta) .
$$

Finally, the modulated NC-QAM/PSK signal $X$ at the RN will be broadcast to the pair of DN1 and DN2.

3) Receiver Design: For the three-timeslot-based $\mathrm{NC}$ scheme, Chen et al. proposed an NC-MRC scheme for combining the network-coded signal and the original signal of the source [13]. At the receiver side, we apply our NC-MRC detection scheme for processing $Y_{i}$. The Viterbi decoding algorithm will be invoked for signal reconstruction. We will then get $\hat{W}_{1}$ and $\hat{W}_{2}$.

\section{ENCODER AND DECODER}

Based on the structure design of TC-NCM conceived in the 367 previous section, we will then focus our attention on designing 368 the encoding algorithm and the transmission mechanism, where 369 Sections IV-A and B outline our motivation and set partitioning 370 philosophy, respectively. In Section IV-C, we design the trans- 371 mission mechanism and coding algorithm, while Section IV-D 372 details our decoder design.

\section{A. Motivation for the Coding Design}

As a joint channel coding and modulation scheme, TCM constitutes a signal-space code, which employs an expanded signal constellation for the sake of absorbing the channel coding parity bits used for providing an error correction capability. In one way, Ungerboeck's TCM scheme uses multilevel/phase signal modulation and simple convolutional coding combined with set-partitioning-based bit-to-symbol mapping [23], [24]. Therefore, the TCM scheme improves the maximum free ED. Yet, in another way, our NCM technique relies on the specific set-partitioning philosophy of [13]. Amalgamating the above two designs results in our TC-NCM coding design, which intrinsically incorporates NC into the classic TCM.

The major differences between classic TCM and our amalgamated scheme are as follows:

1) TCM now operates in a DF-TWR scenario instead of the single-link-based peer-to-peer transmission of [17]. To achieve the desired channel coding gain of TCM, we have to jointly design the coding scheme for the coupled pair of downlinks.

2) Specific constraints are imposed on both the downlink component encoders because the coded-rates are decided by the SNRs $\gamma_{1}$ and $\gamma_{2}$. Furthermore, a moderate SNR-loss is imposed by NC-QAM.

\section{B. Set-Partitioning-Based TC-NCM Design}

As we defined in the previous section, two message sequences 398 at the $\mathrm{RN}$ are $W_{1}$ and $W_{2}$, as shown in Fig. 3. The mode 399 
switching modules select $M_{1}$ and $M_{2}$ constellation modes ${ }^{4}$ to transmit $W_{1}$ and $W_{2}$ subject to the pair of instantaneous SNRs $\gamma_{1}$ and $\gamma_{2}$, separately. Aiming at maximizing the minimum ED of the legitimate symbols transmitted both for $\mathrm{RN} \rightarrow \mathrm{DN} 1$ and $\mathrm{RN} \rightarrow \mathrm{DN} 2$ so as to satisfy the BER constraints, we then conceive the general coding principle of TC-NCM in Algorithm 1. Previous message sequences $W_{1}$ and $W_{2}$ will be mapped into the single symbols of $X_{i}\left[\xi_{i}\right]$ using the set-partitioning-based TC-NCM method of Algorithm 1. What calls for special attention is that 1) Algorithm 1 guarantees that the EDs of both the legitimate symbols of $M_{1}$ and $M_{2}$ for the message $W_{1}$ and $W_{2}$ are always maximized and 2) that the set of all legitimate $\mathrm{NCM}$ symbols is exactly the same as $M_{2}$.

As mentioned above, in conventional systems, the decoding and demodulation are designed separately, whereas TCM provides a solution to integrate the decoding and demodulation. Taking advantage of this property, we combine the NCM technique with TCM, resulting in our proposed TC-NCM algorithm. Based on this architecture, an improved Viterbi decoding algorithm is conceived for decoding the TC-NCM signal at DNs as shown in Algorithm 2, with explanations of its parameters listed as follows:

1) $D_{i}(U, M)$ : the ED of signals between two constellation points;

2) $C_{p, q}{ }^{\prime}$ : the state of coding memory transferring $i$ to $j$;

3) $B M_{i, t}(p, q), q=1,2, \ldots, Q$ : the minimum ED among the $n$th time slot of the received signal $Y_{i, t}$ and $C_{p, q}{ }^{\prime}$;

4) $P M_{i, t}(q)$ : the minimum ED among the candidate sequence and the received sequence, when at the time instant $T$ it has a trellis state of $q$;

5) $S U R_{i, t}(q)$ : the maximum likelihood decoded sequence, when at the time instant $t$ it has a trellis state of $q$.

Relying on the philosophy of TC-NCM conceived above, specific modulation schemes will be proceeded in detail, namely TC-NC-QAM/PSK. Based on the adaptive NC-QAM/PSK schemes of [21] and on the peer-to-peer-coded MQAM design of [17], we will further investigate the joint channel coding and adaptive TC-NCM design for the downlink of DF-TWR. Let us now apply the general method of coded modulation proposed above for the NC-QAM/PSK. The design of the encoding and decoding design constitutes the foundation of the adaptive TC-NC-QAM/PSK scheme, which will be described soon.

\section{Encoder Design of TC-NC-QAM/PSK}

Let us first consider the concrete TC-NC-QAM/PSK coding design based on Figs. 3 and 4 and Algorithm 1. In the following discussion, we will take TC-NC-8PSK and TC-NC-16PSK modulator with code rates of Rate $_{1}=2 / 3$ and Rate $_{2}=3 / 4$ as the specific example to elaborate the general principle of Algorithm 1. In particular, assumption A4) is applied for PSK and/or QAM, which signifies the simplest scenario associated with the former being a subset of the latter. Based on Algorithm 1, the modulated NC symbol is generated by following these steps.

\footnotetext{
${ }^{4}$ In the following discussion, we assume $M_{2} \geq M_{1}$, then each subset consists of $M_{1}$ symbols having the maximum symbol distances.
}

Algorithm 1: Joint Coding-Modulation Algorithm of TC-NCM.

Input: Message $W_{i}, i=1,2, \mathcal{M}_{i}, i=1,2$

Output: TC-NCM symbol $X$

Initial: Determine $k_{1}, k_{2}, n_{1}$ and $n_{2}$ from $M_{1}$ and $M_{2}$.

Step 1: Process $M_{i}$

- Select $M_{i}$ to transmit $W_{i}$

- Compare $M_{i}$ (we assume $M_{2}>M_{1}$ for following use) - If $M_{2} \geq M_{1}$, select $M_{2}$ for follow-up use

- Else select $M_{1}$ for follow-up use

Step 2: Convert the serial signal $W_{2}$ into the parallel signal $n_{2}$

- Operate on $k_{2}$ uncoded data bits of $n_{2}$ to produce $\left(k_{2}+r_{2}\right)$ coded bits

- Partition $M_{2}$ into $2^{k_{2}+r_{2}}$ subsets labeled as $\chi_{2}$, relying on the TCM set partitioning philosophy

Step 3:

- Use the coded bits $\left(k_{2}+r_{2}\right)$ to choose one of the subsets from $\chi_{2}$

- Label the selected subsets as $\chi_{2}^{\left(m_{2}\right)}$, with the symbols

Step 4: in $\chi_{2}^{\left(m_{2}\right)}$ as $\left\{X_{2}^{\left(m_{2}\right)}[0], \ldots, X_{2}^{\left(m_{2}\right)}\left[2^{n_{2}-k_{2}}-1\right]\right\}$

- Operate on the $\left(n_{2}-k_{2}\right)$ additional uncoded bits to choose one of the signal points $X_{2}\left[\xi_{2}\right]$ in $\chi_{2}^{\left(m_{2}\right)}$

- Record the size of subset $\chi_{2}^{\left(m_{2}\right)}$ and the index $\xi_{2}$ of the point $X_{2}\left[\xi_{2}\right]$

Step 5:

- Let $M_{1}$ be a set of $\chi_{2}^{(\backslash)}$

- Generate $\chi_{1}^{\left(m_{1}\right)}, X_{1}\left[\xi_{1}\right]$ and the index $\xi_{1}$ by taking similar Steps 2-4

Step 6: Applying NCM

- Obtain $\xi_{1}$ and $\xi_{2}$

- Generates TC-NCM symbol by $X=X_{2}^{\left(m_{2}\right)}\left[\xi_{1}+\xi_{2}\right.$ $\left.\bmod M_{1}\right]$

Step 1: The message sequences $W_{1}$ and $W_{2}$ at $\mathrm{RN}$ will be 452 converted into parallel signals by the serial-parallel converter, 453 respectively. The serial sequence $W_{2}$ will be addressed first 454 because it is assumed $M_{2} \geq M_{1}$ in Algorithm 1 as an example. 455 The resulting parallel bits are labeled as $c_{i}, i=0,1,2, \ldots, n_{2}, \quad 456$ as shown in Fig. 3. Then, $k_{2}$ bits will be processed by the 457 convolutional encoder of Fig. 4, while $r_{2}$ uncoded bit will be 458 used for constellation mapping. For example, as $\mathrm{RN} \rightarrow \mathrm{DN} 2$ link 459 employs TC-NC-16PSK using Rate $_{2}=3 / 4$, thus the first three 460 bits of $W_{2}=$ "01101001 ..." will be labeled as $c_{2}=0, c_{1}=1, \quad 461$ and $c_{0}=1$, with $k_{2}=2$ and $r_{2}=1$ corresponding to the eight- 462 state TCM.

Step 2: $k_{2}$ uncoded bits are generated by the convolutional encoder to produce three encoded bits. Similar to the classic TCM structure depicted in Fig. 4, in most instances, we employ a rate $\hat{k} /(\hat{k}+1)$ convolutional encoder according to Ungerboeck's design [24]. Continuing by the above example, the bits $\left\{c_{1}, c_{0}\right\}$ are encoded by the system's recursive convolution code to generate $\left\{b_{2}, b_{1}, b_{0}\right\}$. As for the rest of the bits, $\left\{c_{2}\right\}$ 


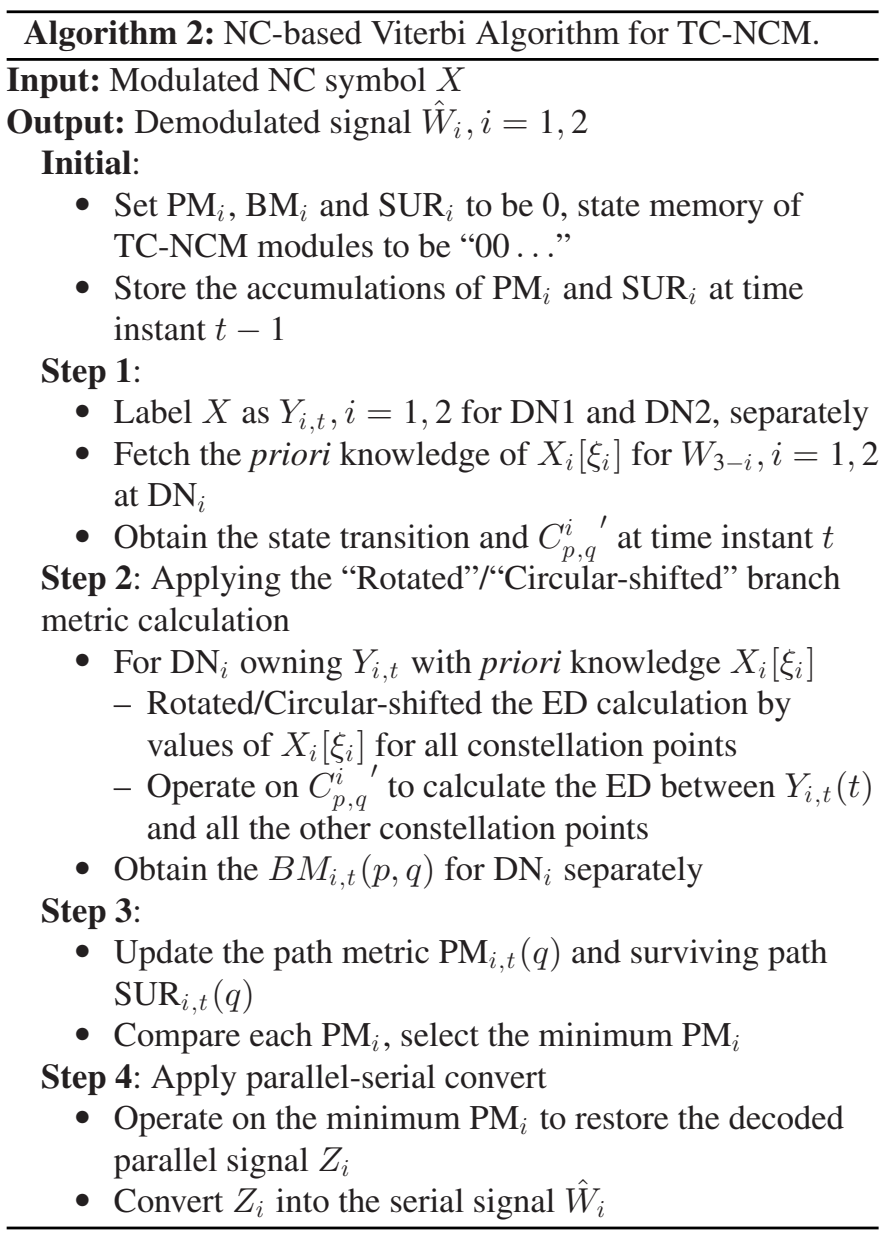

generates $\left\{b_{3}\right\}$, as seen in Fig. 4. Then, we obtain the codeword $C_{1}=\left\{b_{3}, b_{2}, b_{1}, b_{0}\right\}$, with a code rate of Rate $=3 / 4$.

Step 3: According to the general set-partitioning method of TCM, the "Coset Selector 2" of Fig. 3 uses $\left\{b_{2}, b_{1}, b_{0}\right\}$ to choose a subset of the $M_{2}=16$ constellation. At the same time, the "Signal Point Selector 2" uses the bits $\left\{b_{3}\right\}$ to select a specific constellation point from the selected subset. Then, the "Constellation Map 2" of Fig. 3 maps the codeword $C_{1}$ to the selected point of the $M_{2}$ constellation. Fig. 5(a) shows the mapping philosophy of TC-NC-16PSK. The above example $\left\{c_{2}, c_{1}, c_{0}\right\}$ is eventually mapped to $C_{1}=\{0100\}$. Additionally, we may infer the signal's phase of $\theta_{2}=3 \pi / 4$, whereas for QAM we would similarly obtain the symbol's amplitudes.

Step 4: Algorithm 1 and assumption A4 $)^{5}$ guarantee that $M_{1}$ itself is a subset of $M_{2}$, therefore we may employ Steps 1-3 to map $W_{1}$ to a specific symbol of the constellation $M_{1}$. For example, for the $\mathrm{RN} \rightarrow \mathrm{DN} 1$ link employing TC-NC-8PSK for $W_{1}=$ "010011 ..," $\left\{a_{1}, a_{0}\right\}=\{01\}$ will generate $\{010\}$. Thus, we may infer the code rate of $2 / 3$ and the phase $\theta_{1}=\pi / 2$ of the point, which is shown as an example in Fig. 6(a).

Step 5: Use the modulo addition of the phases $\theta_{1}$ and $\theta_{2}$ (or the amplitudes $a^{I}, a^{Q}$ for QAM) to produce the NC symbol phase of $\theta=\left[\theta_{1}+\theta_{2}\right] \bmod 2 \pi$. The concrete operation

${ }^{5}$ This special case of $M_{2} / M_{1}=\mathbb{N}$ or $M_{1} / M_{2}=\mathbb{N}$ is employed in order to simplify the design for QAM/PSK.

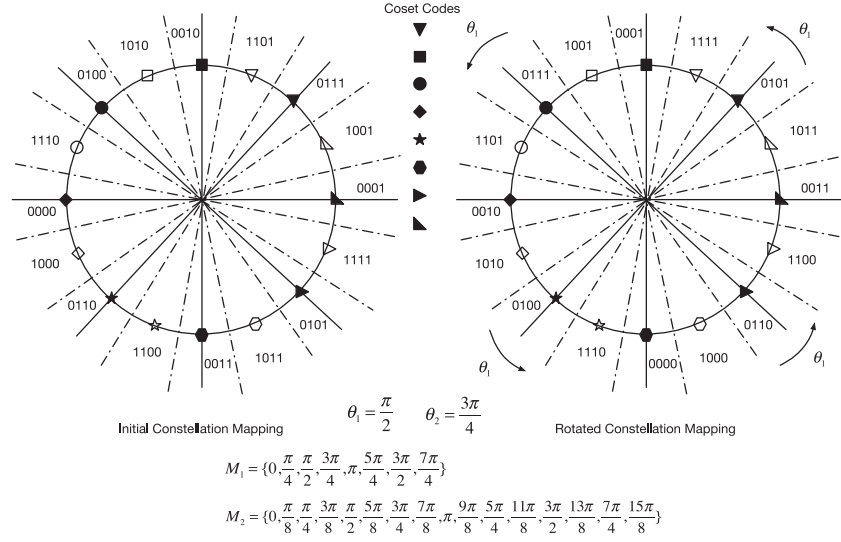

(a)

Fig. 5. Mapping rule of set-partitioning-based TC-NC-16PSK. (a) Coding design and (b) decoding design.

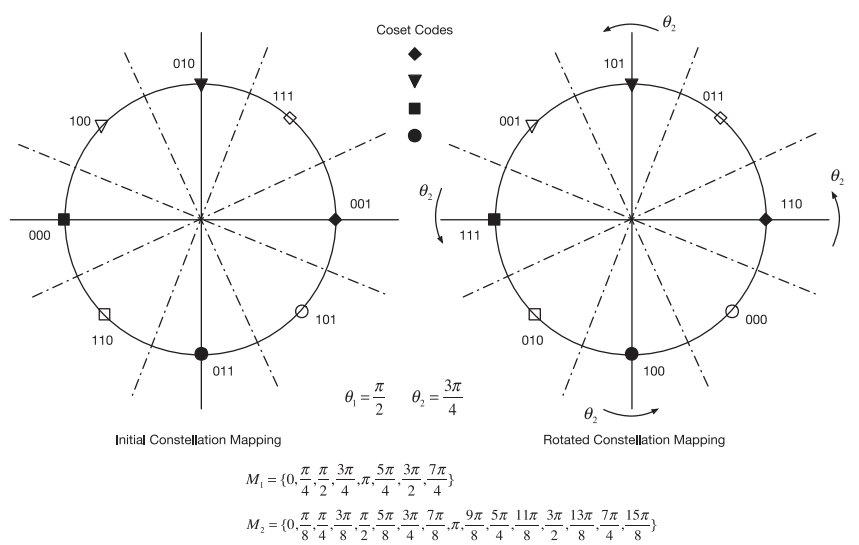

(a)

(b)

Fig. 6. Mapping rule of set-partitioning-based TC-NC-8PSK. (a) Coding design and (b) decoding design.

of our NC-QAM/PSK is detailed in the previous section (see Section III). Continuing the above example, it will generate a phase of $\theta=5 \pi / 4$ along with the corresponding code words $\{0110\}$, converting the signal point into a complex signal. Then, the downlink transmitter of the RN broadcasts the modulated signal $X$ to both DN1 and DN2.

A few further points have to be noted: 1) The trellis may have four, eight, 16, or even more states. In reality, for most applications, the complexity constraints of the current hardware designs typically limit the number of trellis states. 2) Embedding the message $W_{1}$ into $W_{2}$ does not affect the maximum of the minimum ED amongst the legitimate symbols, which implies that beneficial coding gains can be obtained.

\section{Decoder Design of TC-NC-QAM/PSK}

Specifically, our decoder design improves the traditional Viterbi algorithm by invoking the NC philosophy for calculating the metrics as described below. In the demodulator design of NC-QAM/PSK [13], the receivers DN1 and DN2 know $\theta_{i}, i=1,2$ (or $a_{i}^{I}+j a_{i}^{Q}$ for QAM) as a priori and use them to detect the symbol by appropriately rotating (or circularly shifting) the decision region of the MPSK/MQAM constellation. 


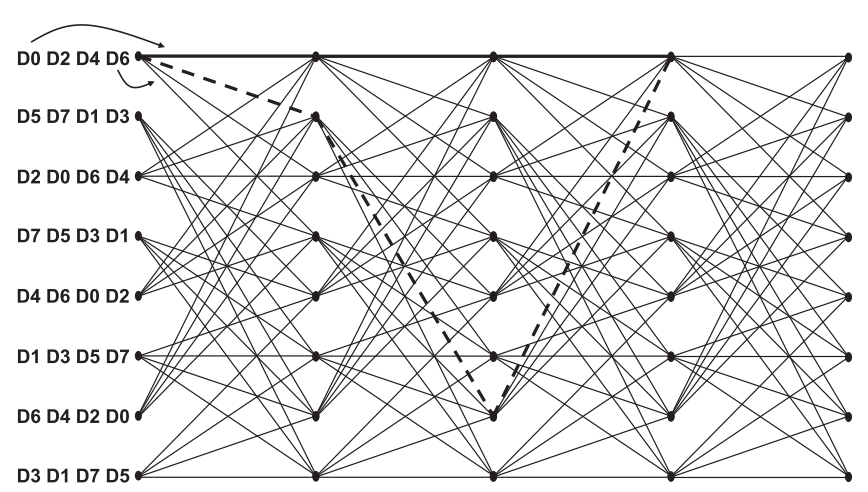

Fig. 7. One possible error path in TC-NC-16PSK (eight-state trellis).
More explicitly, we carry out the above-mentioned circular constellation rotation when calculating the branch metric (BM) in the Viterbi algorithm. This is essential because there is no need to add dedicated modules for separately demodulating the symbols in our current design. The joint demodulation and decoding constitutes an inseparable core of the TCM structure.

We continue our receiver design based on Algorithm 2 on the previous example of using TC-NC-16PSK and TC-NC-8PSK for elaboration. After DN1 and DN2 receive a series of the RN's broadcast signal $X$, the decoders decode the signal using the steps listed below.

Step 1: Let us denote the sequence transmitted by the RN as $X_{0}, X_{1}, X_{2}, \ldots, X_{t}$ and the message sequence received by DN1 and DN2 by $Y_{i, 0}, Y_{i, 2}, Y_{i, 3}, \ldots, Y_{i, t}, i=1,2$, where $t$ is the time instant. We then construct both the specific state diagram and state-transition diagram, corresponding to the convolutional encoder. Fig. 7 shows the eight-state state-transition diagram of 16PSK.

Step 2: As $W_{1}$ has to be transmitted via the $\mathrm{RN} \rightarrow \mathrm{DN} 2$ link, we can identify in advance the specific state transitions based on the constellation mapping rules and on the state-transition diagram. Continue with the Step 1 above, we then calculate the EDs between the received signal and all the other legitimate signal constellation points. Here, the EDs (or BM) are calculated by taking into account the a priori knowledge of having $\theta_{2}=3 \pi / 4$ at the DN2, which is equivalent to pairing the codewords and the modulated phase rotating the constellation anticlockwise by an angle of $\theta_{2}$, as seen in Fig. 6(b). We may therefore obtain minimum $\mathrm{ED} \mathrm{BM}_{2, t}(p, q)$.

Step 3: Update the patch metric, continuing now with the TC-NC-16PSK example. Let us assume all the $P$ states before the current $q$ state are

$$
p(1, q)=q_{1}, \ldots, p(p, q)=q_{p}, \ldots, p(P, q)=q_{P} .
$$

Calculate the sum of each previous path metric $\mathrm{PM}_{2, t-1}$ $\left(q_{p}\right), p=1, \ldots, P$ with the current branch metric $\mathrm{BM}_{2, t}(p, q)$. Find the max one, and let it be the patch metric at current time instant $t$ by calculating

$$
\mathrm{PM}_{2, t}(q)=\max \left\{\mathrm{PM}_{2, t-1}\left(q_{p}\right)+\mathrm{BM}_{2, t}(p, q)\right\}
$$

551 where we have $q=1, \ldots, P$, with $P$ denotes previous $P$ status.
Step 4: Update the surviving path $\operatorname{SUR}_{2, t}(q)$. Each state along 552 the surviving path is associated with an information symbol 553 output during the $t$ th time slot. Compare all the accumulated 554 path metrics $\mathrm{PM}_{2}$ that merge into the same state and then retain 555 the more likely one, while discarding the other one. Thus, the 556 decoding output is uniquely and unambiguously specified by 557 the surviving path $\mathrm{SUR}_{2, t}$ (see dashed line in Fig. 7), having the 558 minimum accumulated path metric along all the states. A final 559 note about this step is that when employing for judgment at each 560 state, there may occur that the ED values of two paths are the 561 same, then any of the two path may be used for decoding the 562 sequence because the accumulated path values are the same.

Step 5: Convert the decoded parallel signals, say, $\left\{\hat{c}_{2}, \hat{c}_{1}, \hat{c}_{0}\right\} \quad 564$ into a serial sequence. Then, DN2 recovers the received message 565 $\hat{W}_{1}$. For the $\mathrm{RN} \rightarrow \mathrm{DN} 1$ link, we may employ the same method 566 and the a priori information $\theta_{1}$, as in the previous steps, to 567 recover the message $\hat{W}_{2}$.

\section{Performance EVAluation}

Upon using the subset partitioning inherent in coded modulation, trellis or lattice codes designed for fading channels can be directly amalgamated with adaptive modulation. Therefore, we can adjust both the power and the transmit rate (constellation size) as a function of the instantaneous SNR without affecting the attainable channel coding gain. The relationships between power, rates, and BER constraints ${ }^{6}$ were investigated in detail in [16] and [21]. More explicitly, a transmit power control policy of joint power- and rate-adaptive NCM designed for DF-TWR's downlink was derived in [21]. If we use this power control policy in conjunction with the superimposed trellis code proposed in the previous section (see Section IV), then we can reduce the transmit power $S\left(\gamma_{1}, \gamma_{2}\right)$ by the effective power gain of $G_{e}$ of TCM and still maintain the target BER. Some of the coding gains of TCM-PSK and TCM-QAM are summarized in Tables I and II [23], where $\tilde{m}$ denotes the number of input uncoded bits, and $h_{0}, h_{1}$, and $h_{2}$ are parity-check coefficients [23]. These gains can be achieved in each mode of our adaptive TC-NC-QAM/PSK scheme.

We will next get into the performance analysis in more detail. Before presenting the analysis, we list all the symbols and their meaning in Tables III and IV to augment our exposition.

In discrete-rate adaptive NCM design, we determine the constellation size associated with each SNR by discretizing the range of channel fade levels. Specifically, the range of $\gamma_{i}$ will be divided into $N_{i}$ fading regions, where $\mathcal{R}_{i, n_{i}}=\left[\gamma_{i, n_{i}-1}, \gamma_{i, n_{i}}\right)$, $n_{i}=1, \ldots, N_{i}$ denotes the fading boundaries, where we have $\gamma_{i, 0}=0, \gamma_{i, N_{i}}=\infty$. We, hence, activate the pair of fixed constellation sizes $M_{1, n_{1}}, M_{2, n_{2}}$, when we have $\gamma_{1} \in \mathcal{R}_{1, n_{1}}, \gamma_{2} \in$ $\mathcal{R}_{2, n_{2}}$. For discrete-rate adaptive NCM, we denote the discrete constellation sizes by $M_{1, \eta}, \eta=1,2, \ldots, n_{1}$ and $M_{2, \delta}, \delta=$ $1,2, \ldots, n_{2}$, as shown in Fig. 8. We define in Table III that the BER constraints for $\mathrm{RN} \rightarrow \mathrm{DN} 1$ and $\mathrm{RN} \rightarrow \mathrm{DN} 2$ be $P_{1}$ and $P_{2}$. Then, based on BER bounds in [16] and [21] and on coding

\footnotetext{
${ }^{6}$ In general the desired value of the ED $d_{0}$ is determined from the target BER of the system.
}

\section{. . . , . , . .

(t)

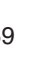
. . 
TABLE I

CHANNEl CODING GAIN OF TCM-PSK

\begin{tabular}{|c|c|c|c|c|}
\hline \multirow[t]{2}{*}{ Constellation Size } & \multicolumn{3}{|c|}{ Number of Coding bits State Coding Gain (dB) } & \multirow{2}{*}{$\frac{H(D)}{h_{0} h_{1} h_{2}}$} \\
\hline & & & & \\
\hline 4PSK/8PSK $(\tilde{m}=2)$ & 1 & 4 & 3.01 & $0502 \backslash$ \\
\hline 4PSK/8PSK $(\tilde{m}=2)$ & 2 & 8 & 3.60 & 110204 \\
\hline 4PSK/8PSK $(\tilde{m}=2)$ & 2 & 32 & 4.59 & 451634 \\
\hline 16PSK/8PSK $(\tilde{m}=3)$ & 1 & 4 & 3.54 & $0502 \backslash$ \\
\hline 16PSK/8PSK $(\tilde{m}=3)$ & 1 & 8 & 4.01 & $1304 \backslash$ \\
\hline $16 \mathrm{PSK} / 8 \mathrm{PSK}(\tilde{m}=3)$ & 1 & 32 & 5.13 & $4510 \backslash$ \\
\hline
\end{tabular}

TABLE II

ChanNel CoDing GaIn OF TCM-QAM

\begin{tabular}{|c|c|c|c|c|c|}
\hline \multirow[t]{2}{*}{ Constellation Size } & \multirow[t]{2}{*}{ Number of Coding bits } & \multirow[t]{2}{*}{ State } & \multirow[t]{2}{*}{ Coding Gain $(\mathrm{dB})$} & \multicolumn{2}{|c|}{$H(D)$} \\
\hline & & & & $h_{0}$ & $h_{1} \quad h_{2}$ \\
\hline 4QAM $(\tilde{m}=2)$ & 1 & 4 & 3.01 & 05 & $02 \backslash$ \\
\hline $4 \mathrm{QAM}(\tilde{m}=2)$ & 2 & 8 & 3.98 & 11 & 0204 \\
\hline 4QAM $(\tilde{m}=2)$ & 2 & 32 & 4.77 & 41 & $06 \quad 10$ \\
\hline 16QAM $(\tilde{m}=4)$ & 1 & 4 & 3.01 & 05 & $02 \backslash$ \\
\hline 16QAM $(\tilde{m}=4)$ & 2 & 8 & 3.98 & 11 & 0204 \\
\hline 16QAM $(\tilde{m}=4)$ & 2 & 32 & 4.77 & 41 & $06 \quad 10$ \\
\hline 64QAM $(\tilde{m}=5)$ & 1 & 4 & 2.80 & 05 & $02 \backslash$ \\
\hline 64QAM $(\tilde{m}=5)$ & 2 & 8 & 3.77 & 11 & 0204 \\
\hline 64QAM $(\tilde{m}=5)$ & 2 & 32 & 4.56 & 41 & $06 \quad 10$ \\
\hline
\end{tabular}

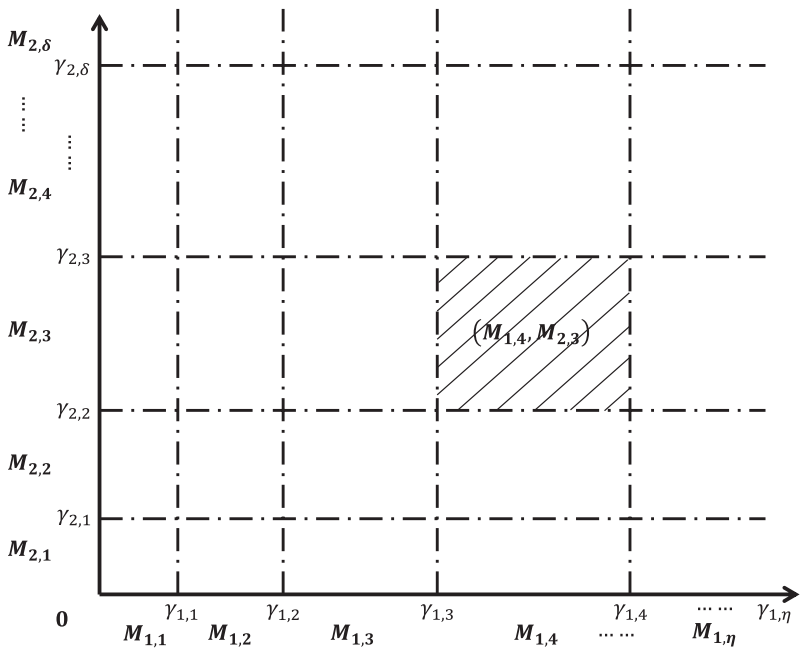

Fig. 8. Fading region zoning.

gain $G_{e}$, we arrive at the pair of BER expression for TC-NCQAM/PSK:

$$
\left\{\begin{array}{l}
P_{1} \leq \beta_{1} \exp \left[\frac{-\beta_{2} G_{e_{\eta}} \lambda_{1} \gamma_{1} \frac{S_{\eta \delta}\left(\gamma_{1}, \gamma_{2}\right)}{S}}{2^{\beta_{3} k_{1, \eta}}-\beta_{4}}\right] \\
P_{2} \leq \beta_{1} \exp \left[\frac{-\beta_{2} G_{e_{\delta}} \lambda_{2} \gamma_{2} \frac{S_{\eta \delta}\left(\gamma_{1}, \gamma_{2}\right)}{S}}{2^{\beta_{3} k_{2, \delta}-\beta_{4}}}\right]
\end{array}\right.
$$

with the transmit rate for the pair of links given by

$$
\left\{\begin{array}{l}
k_{1, \eta}=\frac{\log _{2} M_{1, \eta}}{\beta_{3}} \\
k_{2, \delta}=\frac{\log _{2} M_{2, \delta}}{\beta_{3}} .
\end{array}\right.
$$

Interpretation of parameters are shown in Table III. To facilitate the following discussion, (13) can be rewritten as

607

608

$$
\left\{\begin{array}{l}
M_{1, \eta} \leq \beta_{4}+K_{1} G_{e_{\eta}} \lambda_{1} \gamma_{1} \frac{S_{\eta \delta}\left(\gamma_{1}, \gamma_{2}\right)}{S} \\
M_{2, \delta} \leq \beta_{4}+K_{2} G_{e_{\delta}} \lambda_{2} \gamma_{2} \frac{S_{\eta \delta}\left(\gamma_{1}, \gamma_{2}\right)}{S}
\end{array}\right.
$$

with the SNR-loss $\lambda_{i}{ }^{7}$ denoted by

$$
\left\{\begin{array}{l}
\lambda_{1}=1, \lambda_{2}=\frac{1-M_{2, \delta}^{-1}}{1-M_{1, \eta}^{-1}}, \quad \text { if } M_{1, \eta} \geq M_{2, \delta} \geq 2 \\
\lambda_{1}=\frac{1-M_{1, \eta}^{-1}}{1-M_{2, \delta}^{-1}}, \lambda_{2}=1, \text { if } M_{2, \delta} \geq M_{1, \eta} \geq 2 \\
\lambda_{1}=\lambda_{2}=1, \text { for NC-PSK Scheme }
\end{array}\right.
$$

where $K_{i}=-\beta_{2} / \ln \left(P_{1} / \beta_{1}\right)$ is related to the BER constraints $P_{1}$ and $P_{2}$. Additionally, $\beta_{1}, \beta_{2}, \beta_{3}, \beta_{4}$ are constants that correspond to the specific QAM/PSK modes [26], as shown in Table IV.

Combining our objectives with the constraints of (13), we next formulate the optimization problem.

Problem Definition: Maximizing the weighted achievable throughput, subject to both the average power and BER constraints, yields the following:

$$
\begin{aligned}
\operatorname{maximize} \frac{R}{B}= & \sum_{\eta=1}^{N_{1}} \sum_{\delta=1}^{N_{2}}\left[\omega_{1}\left(k_{1, \eta}-\mathrm{r}_{\eta}\right)+\omega_{2}\left(k_{2, \delta}-\mathrm{r}_{\delta}\right)\right] \\
& \times \int_{\gamma_{1, \eta-1}}^{\gamma_{1, \eta}} p\left(\gamma_{1}\right) d \gamma_{1} \int_{\gamma_{2, \delta-1}}^{\gamma_{2, \delta}} p\left(\gamma_{2}\right) d \gamma_{2}
\end{aligned}
$$

subject to

$$
\left\{\begin{array}{l}
\sum_{\eta=1}^{N_{1}} \sum_{\delta=1}^{N_{2}} \int_{\gamma_{1, \eta-1}}^{\gamma_{1, \eta}} \int_{\gamma_{2, \delta-1}}^{\gamma_{2, \delta}} \frac{S_{\eta \delta}\left(\gamma_{1}, \gamma_{2}\right)}{S} p\left(\gamma_{1}\right) p\left(\gamma_{2}\right) d \gamma_{1} d \gamma_{2}=1 \\
0<\gamma_{1,1}<\cdots<\gamma_{1, \eta-1}<\gamma_{1, \eta}<\cdots<\gamma_{1, N_{1}} \\
0<\gamma_{2,1}<\cdots<\gamma_{2, \delta-1}<\gamma_{2, \delta}<\cdots<\gamma_{2, N_{2}} \\
S_{\eta \delta}\left(\gamma_{1}, \gamma_{2}\right) \geq 0 .
\end{array}\right.
$$

This is a high-dimensional multivariable discrete optimization problem, whose closed-form solution is hard to obtain and is rather difficult to solve with a conventional convex optimization method. Fortunately, inspired by the fading region zoning philosophy of [16] and discrete NCM approaches of [21], we develop the following power control policy based on (15):

$$
\frac{S_{\eta \delta}\left(\gamma_{1}, \gamma_{2}\right)}{\bar{S}}=\max \left\{\frac{M_{1, \eta}-c_{4}}{\lambda_{1} G_{e_{\eta}} K_{1} \gamma_{1}}, \frac{M_{2, \delta}-c_{4}}{\lambda_{2} G_{e_{\delta}} K_{2} \gamma_{2}}, 0\right\} .
$$

A few further points have to be noted about the optimization 625 problem:

1) As is graphically portrayed in Fig. 8, in each fading region, one transmit power $S_{\eta \delta}\left(\gamma_{1}, \gamma_{2}\right)$ corresponds to two rates $\left(M_{1, \eta}\right.$ and $\left.M_{2, \delta}\right)$. However, $M_{1, \eta}$ and $M_{2, \delta}$ destined for DN1 and DN2 cannot reach their optimal match with the $S_{\eta \delta}\left(\gamma_{1}, \gamma_{2}\right)$ at the same time, except when $\gamma_{1}=\gamma_{2}$ which is practically impossible in time-varying fading channels. That is to say, (18) signifies an inevitable power-loss or rate-loss.

\footnotetext{
${ }^{7}$ Here, the SNR loss is imposed by NC-QAM, which implies some extra energy consumption will be resulted due to the direct current (DC) bias [13] The in-depth derivation of the SNR loss is provided in the Appendix.
} 
TABLE III

LIST OF PARAMETER SPECIFICATIONS

\begin{tabular}{llcl}
\hline \hline \multicolumn{1}{c}{ Symbols } & \multicolumn{1}{c}{ Meaning of the symbol } & \\
\hline$G_{e}$ & channel coding gain & & \\
$\tilde{m}$ & uncoded data bits & $S_{\eta \delta}\left(\gamma_{1}, \gamma_{2}\right)$ & transmit power at RN \\
$\bar{S}$ & average transmit power & $k_{1, \eta}, k_{2, \delta}$ & transmit rates \\
$\eta, \delta$ & the subscripts of region zoning & $K_{1}, K_{2}=-\beta_{2} / \ln \left(P_{i} / \beta_{1}\right)$ & the number of zoning \\
$\omega_{1}, \omega_{2}$ & weighting factors of each user & $N_{1}, N_{2}$ & redundant bits per symbol \\
$P_{1}, P_{2}$ & BER constraints & $r_{\eta}, r_{\delta}$ & the boundaries of zoning \\
$M_{1, \eta}, M_{2, \delta}$ & constellation sizes for the $\eta$ th, $\delta$ th area & $\gamma_{1, \eta}, \gamma_{2, \delta}$ & distributions of SNR \\
$\bar{\gamma}_{1}, \bar{\gamma}_{2}$ & average SNRs & $p\left(\gamma_{1}\right), p\left(\gamma_{2}\right)$ & fading region zoning \\
$\beta_{1}, \beta_{2}, \beta_{3}, \beta_{4}$ & BER curve fitting parameters (Table IV) & $\mathcal{R}_{1, n}, \mathcal{R}_{2, n}$, & parity-check coefficients [27] \\
$\lambda_{1}, \lambda_{2}$ & SNR-loss imposed by NC-QAM & $H(D), h_{0}, h_{1}, h_{2}$ & \\
\hline \hline
\end{tabular}

TABLE IV

CONSTANTS IN BER APPROXIMATIONS

\begin{tabular}{lcccc}
\hline \hline & $\beta_{1}$ & $\beta_{2}$ & $\beta_{3}$ & $\beta_{4}$ \\
\hline MQAM & 0.2 & 1.5 & 1 & 1 \\
MPSK(Mode1) & 0.05 & 6 & 1.9 & 1 \\
MPSK(Mode2) & 0.2 & 7 & 1.9 & -1 \\
MPSK(Mode3) & 0.25 & 8 & 1.94 & 0 \\
\hline \hline
\end{tabular}

2) Intuitively, to maximize the pair of user's weighted sum rate is equivalent to find the optimal region partitions $\mathcal{R}_{1, n_{1}}=\left[\gamma_{1, n_{1}-1}, \gamma_{1, n_{1}}\right), n_{1}=1, \ldots, N_{1}$ and $\mathcal{R}_{2, n_{2}}=$ $\left[\gamma_{2, n_{2}-1}, \gamma_{1, n_{2}}\right), n_{2}=1, \ldots, N_{2}$, which are jointly determined by the average power constraint and the fading distribution $p\left(\gamma_{i}\right), i=1,2 . \mathcal{R}_{1, \eta}$ and $\mathcal{R}_{2, \delta}$ cannot be found in a closed form, and hence, it has to be determined using numerical search techniques.

Of particular note is that the search method of this optimization problem imposes an excessive computational complexity due to its multilayer nested loop. The traditional search technique of [16] adopted an exhaustive search (ES) method, which again, imposes an excessive computational complexity. Although the optimization problem can be solved with the aid of the ES algorithm, we have designed a reduced-complexity heuristic algorithm to solve this problem by invoking a simulated annealing (SA) algorithm, as seen in Algorithm 3. Finally, once the optimal boundaries have been obtained, they can be stored in a lookup table, hence dispensing with real-time calculations.

From the above derivation and discussion, it might be concluded that the combination of TC-NCM with discreterate adaptive modulation holds the promise of improving the throughput of DF-TWR, while maintaining the same BER performance. Relying on the optimization problem of (17) and (18), we will next present the analytical and simulation results.

\section{NUMERICAL RESULTS AND ANALYSIS}

In this section, we provide both simulation results and numerical results for characterizing both the BER performance and the bandwidth efficiency of the four-state TC-NCM scheme of Tables I and II described in the previous section. These results are obtained using both the analytical formulas derived in Section $\mathrm{V}$ and simulations. The simulations are based on

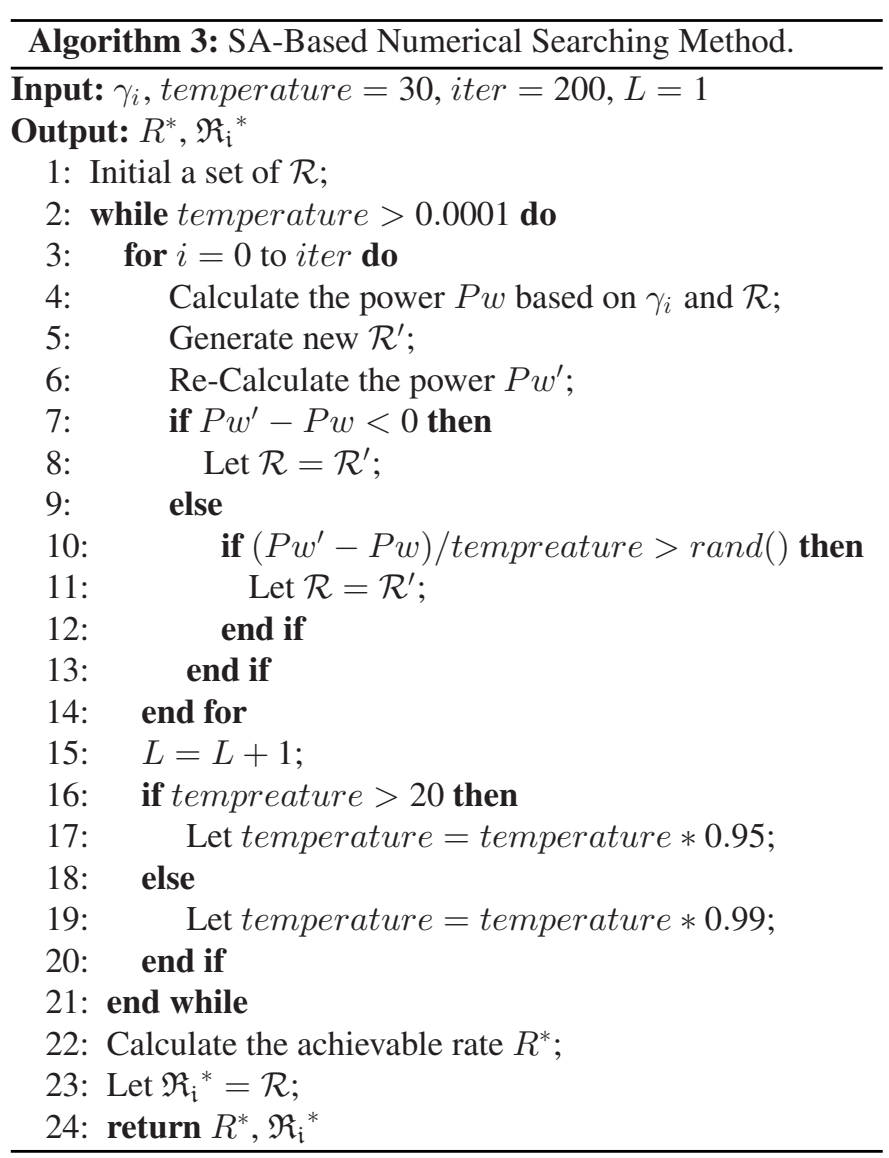

Ungerboeck's TCM method [24], with the NCM embedded into 667 TCM, using the following experimental conditions: 668

1) Common Parameter Settings:

a) Fading distribution: Let the fading channels obey Rayleigh fading adhering to A1). Then, the distribution of $\gamma_{i}$ is given by letting $m=1$ in (1), yielding

$$
p\left(\gamma_{i}\right)=\frac{1}{\bar{\gamma}_{i}} \exp \left(-\frac{\gamma_{i}}{\bar{\gamma}_{i}}\right), i=1,2 .
$$

b) Redundancy rate: Let $r_{\eta}=1$ and $r_{\delta}=1$.

c) Coding rate: We employ a rate of $k /(k+1)$ for the 674 convolutional encoder.

d) TC-NCM state: We employ 4-State, 8-State, and 676 32-State TC-NCM having the coding gains listed in 677 Tables I and II. 


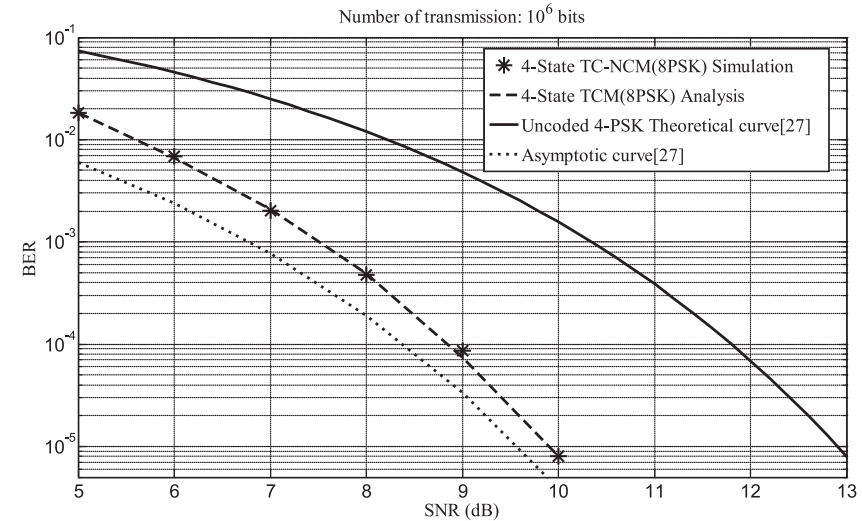

Fig. 9. BER performance of four-state TC-NCM-8PSK.

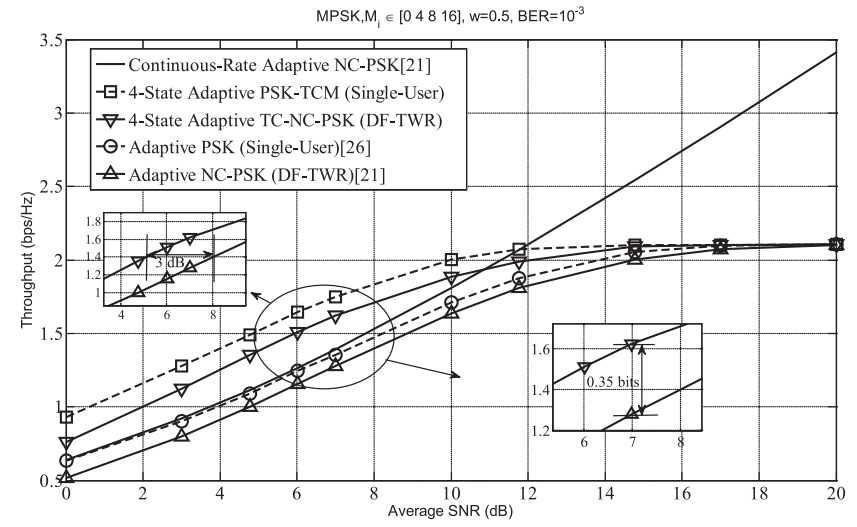

Fig. 10. Throughput of four-state adaptive TC-NC-PSK. e) SNR-loss for NC-QAM: If we let the constellation sets of TC-NC-QAM be $M_{i} \in\{0,4,16,64\}$, then the exact SNR-loss of (16) can be calculated, as shown in Table IV.

f) BER and average power constraint: The target BER is $10^{-3}$ and $\bar{S}=1$.

g) Range of SNR fluctuations: We restrict the nearinstantaneous SNR fluctuations to limited dynamic range, which was set to be ten times the average SNR. ${ }^{8}$

h) BER parameters $\beta_{i}, i=1,2,3,4$ : MQAM and MPSK (Mode 1).

i) Weighting factors: Let $\omega_{i}=0.5, i=1,2$.

2) Fig. 9 Parameter Settings:

a) Constellation size for the $\mathrm{RN} \rightarrow \mathrm{DN} 1$ link: $\mathrm{TC}-\mathrm{NCM}$ with $M_{1}=8$.

b) Asymptotic curve and uncoded theoretical four-PSK curve [27].

c) TC-NCM State: Four-State TCM.

d) Number of transmission bits: $10^{6}$.

3) Fig. 10 Parameter Settings:

a) Continuous-rate adaptive NC-PSK scheme: [21, Eq. (40)].

\footnotetext{
${ }^{8}$ This is a reasonable choice for Rayleigh fading channels. For example, we may have the fluctuations of instantaneous SNR to be $\gamma_{i} \in\left[0,10 \bar{\gamma}_{i}\right], i=$ 1,2. Therefore, the probability of instantaneous SNR beyond $10 \bar{\gamma}_{i}$ is about $4.5400 \times 10^{-5} / \bar{\gamma}_{i}$, which is small enough to be neglected.
}

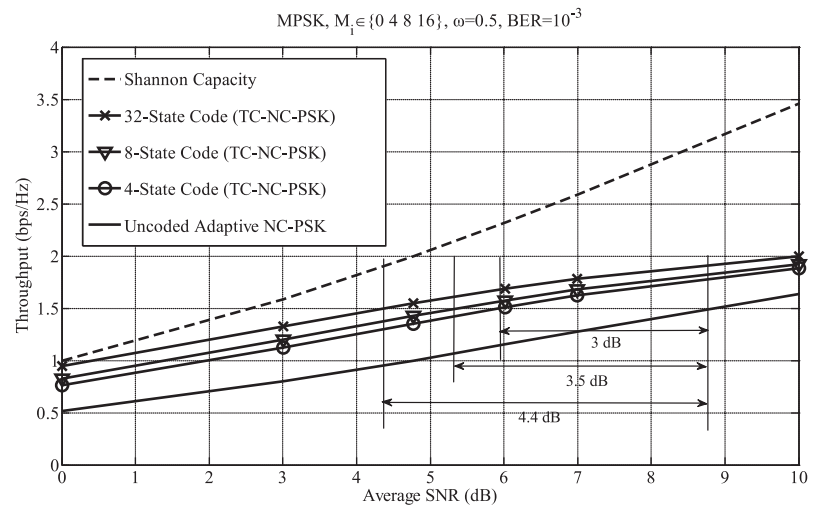

Fig. 11. Throughput versus average SNR for different number of states in TC-NC-PSK.

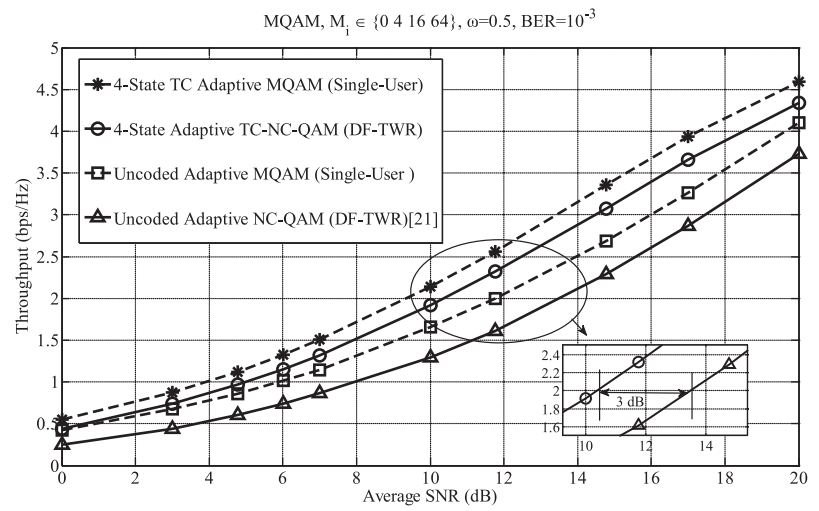

Fig. 12. Throughput of four-state adaptive TC-NC-QAM.

TABLE V

SNR-LOSS OF TC-NC-QAM

\begin{tabular}{lccc}
\hline \hline & 4QAM & 16QAM & 64QAM \\
\hline 4QAM & $\lambda=1$ & $\lambda=4 / 5$ & $\lambda=16 / 21$ \\
16QAM & $\lambda=4 / 5$ & $\lambda=1$ & $\lambda=20 / 21$ \\
64QAM & $\lambda=16 / 21$ & $\lambda=20 / 21$ & $\lambda=1$ \\
\hline \hline
\end{tabular}

b) Adaptive PSK scheme: [26, ch. 9.4.2, 702 Eqs. (9.61)-(9.67)].

c) Discrete-rate adaptive NC-PSK scheme: $[21$, Sce- 704 nario 6, Tab. II].

d) Constellation size $M_{i} \in\{0,4,8,16\}$.

4) Fig. 11 Parameter Settings:

a) TC-NCM State: 4-State, 8-State, 32-State TCM.

b) Constellation set: $M_{i} \in\{0,4,8,16\}$.

c) Channel coding gains: Column 4 of Table I.

5) Fig. 12 Parameter Settings:

a) Adaptive NC-QAM: [21, Eq. (39)]. Eqs. (9.19)-(9.22)].

c) Constellation set: $M_{i} \in\{0,4,16,64\}$. 715

d) TC-NCM State: Four-State TCM. 716

e) Channel coding gains: Column 4 of Table II. $\quad 717$

f) SNR-loss: Table V. 718

A range of representative numerical results are presented for 719 validating our previous analysis. Let us first demonstrate that 720 
embedding NCM into TCM does not affect the channel coding gain, and that the resultant TC-NCM design has the same BER performance as TCM. Fig. 9 plots our BER simulation results for the $\mathrm{RN} \rightarrow \mathrm{DN} 1$ link based on our TC-NC-PSK scheme. For comparison, the benchmarks include the BER simulation results of four-state TCM-8PSK, the uncoded 4PSK, as well as the asymptotic performance of TCM-PSK [27, Fig. 4]. Observe that the TCM-8PSK curve and our proposed TC-NC-8PSK curve match well with each other, which confirms that embedding NCM into TCM does not affect the BER performance. Hence, it can be concluded that we can exploit the coding gain of TCM for adaptive NCM. For example, four-state TCM offers a coding gain of $3.01 \mathrm{~dB}$ [23], compared to uncoded QPSK. Therefore, our TC-NCM also obtains the same coding gain with the aid of four-state TCM. Higher gains may be obtained if we adopt a higher memory TCM design. Based on this conclusion, we could further analyze the performance of TC-NCM.

We plot the achievable throughput of the four-state TC-NCPSK in Fig. 10, where the benchmarks are the achievable rate of the single-user adaptive TCM, the continuous-rate adaptive NC-PSK and the discrete-rate adaptive TC-NC-PSK schemes. It can be observed that the proposed TC-NC-PSK is superior to its counterparts operating without TCM, where the throughput gain ranges from 0.25 to 0.35 bits/symbol at low SNRs. We will offer further observations during our forthcoming discourse.

1) It can be concluded that our proposed TC-NC-PSK attains a higher throughput than adaptive NC-PSK [21] for the DF-TWR's downlink. The achievable rate of TC-NCPSK approaches the single-user TCM scheme's performance, despite the fact that our scheme supports the more challenging scenario of DF-TWR.

2) The system benefits substantially from channel coding (by about $3 \mathrm{~dB}$ ), when the average SNRs are low, whereas it only benefits modestly at high average SNRs. This is due to the fact that upon increasing the average SNR, the BER target of $10^{-3}$ can be readily satisfied. Therefore, the channel coding benefits become modest. This is the rationale of gradually increasing the code-rate toward unity.

3) It is also worth noting that the throughput of the discreterate adaptive scheme saturates upon increasing the average SNR at the same value (about $2.105 \mathrm{bps} / \mathrm{Hz}$ ) as that of its uncoded adaptive NC-PSK counterpart. This is due to the fact that in (14), we adopt $\beta_{3}=1.9$ for MPSK. Hence, for the optimal solution, we have an achievable rate of $\left(\log _{2} 16\right) / 1.9 \approx 2.1053$.

We then further present the throughput of adaptive TC-NCPSK for higher complexity codes in Fig. 11. Naturally, a higher number states will offer a higher coding gain ( $4.5 \mathrm{~dB}$ or more) but will increase the decoding complexity of the design.

In order to complete our adaptive TC-NCM design, we characterize the attainable throughput of TC-NC-QAM in Fig. 12. Similar trends prevail as previously. Of particular note is that our TC-NCM scheme adopts a joint coding and modulation design, which reduces the associated hardware cost. Therefore, it is suitable for diverse practical applications. Based on Figs. 10-12, it can be concluded that our holistic design has the advantage of an improved adaptability and high throughput, especially for transmission at low average SNRs.

\section{CONCLUSION}

In this paper, we developed a transmission regime for the downlink of a DF-TWR system, relying on the combination of TCM and NCM. The general principle of combining coset codes with NCM was presented. We then conceived the transmitter structure of our TC-NCM scheme and applied this design to practical QAM/PSK arrangements. We continued by proposing the general encoding and decoding algorithm for TC-NC-QAM/PSK, where our transmission mechanism was interpreted with the aid of examples. Finally, the attainable performance of our discrete-rate adaptive NCM scheme was investigated. Our simulation and numerical results indicate that compared to uncoded adaptive NC-QAM/PSK and to peerto-peer adaptive modulation, our proposed TC-NCM schemes are capable of further improving the throughput of DF-TWR systems while maintaining the same BER performance. For future studies, an attractive direction is to investigate the attainable shaping gain of the constellation, which may further improve the system's throughput.

\section{APPENDIX \\ DERIVATION OF THE SNR-LOSS [13]}

An SNR-loss is imposed by NC-QAM when the transmit rates for the pair of downlinks of TWR are different. This implies that for the coupled $\mathrm{RN} \rightarrow \mathrm{DN} 1$ and $\mathrm{RN} \rightarrow \mathrm{DN} 2$ links, if one of the user's rate and power achieves the optimal match, ${ }^{9}$ the other one will have a rate determined by the maximum constellation size it can employ.

Derivation of the SNR-loss: Assume that $M_{1}$ and $M_{2}\left(M_{2}>\right.$ $\left.M_{1}\right)$ are the constellation sizes for the $\mathrm{RN} \rightarrow \mathrm{DN} 1$ and $\mathrm{RN} \rightarrow \mathrm{DN} 2$ links, respectively. By exploiting the symbol error rate (SER) formula of NC-QAM [13], the SER of a circularly shifted MQAM constellation is identical to that of the original MQAM for the same minimum symbol distance.

According to (5) and (6) and exploiting that $a_{i}^{I}, a_{i}^{Q} \in \mathcal{A}_{i}$, we may obtain the minimum ED of the symbols

$$
\left\{\begin{array}{l}
d_{1}=\left(\sqrt{M_{2}} / \sqrt{M_{1}}\right) d \\
d_{2}=d
\end{array}\right.
$$

with $d$ denoting half of the symbol distance in QAM. We have the SER formulated as

$$
\operatorname{SER}_{i}=\frac{4\left(\sqrt{M_{i}}-1\right)}{\sqrt{M_{i}}} Q\left(\sqrt{\frac{\left|h_{i}\right|^{2} d_{i}^{2}}{N_{0} / 2}}\right), i=1,2 .
$$

Let us insert $d_{1}$ as well as $d_{2}$ into (21) and introduce 815 the $M_{1}$ - and $M_{2}$-dependent coefficient of $\lambda_{i}=\left(1-M_{i}^{-1}\right) / 816$ $\left(1-M_{2}{ }^{-1}\right)$. Then, we may arrive at the unified SER expres- 817 sions of NC-QAM, given by

$$
\operatorname{SER}_{i}=\frac{4\left(\sqrt{M_{i}}-1\right)}{\sqrt{M_{i}}} Q\left(\sqrt{\frac{1.5 \lambda_{i} \gamma_{i}}{M_{i}-1}}\right), i=1,2 .
$$

\footnotetext{
${ }^{9}$ Here, the optimal match means that the transmit power is the one which

happens to be the power that a specific modulation mode requires.
} 
Since $M_{2}>M_{1}$, we have $\lambda_{1}<1$ and $\lambda_{2}=1$, which implies imposing an SNR loss for the $\mathrm{RN} \rightarrow \mathrm{DN} 1$ link that remains constant across the entire SNR range.

Analysis: The reason for this SNR loss at the receiver of DN1 can be stated as follows. Since QAM is regarded as a pair of orthogonal pulse-amplitude modulation (PAM) signals, we may simply focus our discussions on the $I$ component. Given $a_{2}^{I}$, the legitimate symbols at the DN1 have a nonzero mean of

$$
d\left[2 \sqrt{M_{2}}\left(a_{2}^{I} \bmod \frac{1}{\sqrt{M_{1}}}\right)+1-\frac{\sqrt{M_{2}}}{\sqrt{M_{1}}}\right] .
$$

In contrast to the classic zero-mean $\sqrt{M_{1}}$-ary PAM, the direct current bias of such a circularly shifted $\sqrt{M_{1}}$-ary PAM constellation will result in some extra energy consumption, which therefore results in the above-mentioned SNR loss.

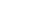

[1] R. Ahlswede, N. Cai, S. Li, and R. W. Yeung, "Network information flow," IEEE Trans. Inf. Theory, vol. 46, no. 4, pp. 1204-1216, Jul. 2000.

[2] S. Y. Li, R. W. Yeung, and N. Cai, "Linear network coding," IEEE Trans. Inf. Theory, vol. 49, no. 2, pp. 371-381, Feb. 2003.

[3] Y. Wu, P. A. Chou, and S. Y. Kung, "Information exchange in wireless networks with network coding and physical-layer broadcast," Microsoft Res., Redmond, WA, USA, Tech. Rep. MSR-TR-2004, 2004.

[4] P. Larsson, N. Johansson, and K. E. Sunell, "Coded bi-directional relaying," in Proc. IEEE 63rd Veh. Technol. Conf., Melbourne, VIC, Australia, May 2006, pp. 851-855.

[5] H. V. Nguyen, S. X. Ng, and L. Hanzo, "Performance bounds of network coding aided cooperative multiuser systems," IEEE Signal Process. Lett., vol. 18, no. 7, pp. 435-438, Jul. 2011.

[6] H. V. Nguyen, C. Xu, S. X. Ng, and L. Hanzo, "Non-coherent near-capacity network coding for cooperative multi-user communications," IEEE Trans. Commun., vol. 60, no. 10, pp. 3059-3070, Oct. 2012.

[7] P. Popovski and H. Yomo, "Physical network coding in two-way wireless relay channels," in Proc. IEEE Int. Conf. Commun., Jun. 2007, pp. 707-712.

[8] L. L. Xie, "Network coding and random binning for multi-user channels," in Proc. 10th Can. Workshop Inf. Theory, Jun. 2007, pp. 85-88.

[9] Y. Wu, "Broadcasting when receivers know some messages a priori," in Proc. IEEE Int. Symp. Inf. Theory, Jun. 2007, pp. 1141-1145.

[10] P. Larsson, "A multiplicative and constant modulus signal based network coding method applied to CB-relaying," in Proc. IEEE Veh. Technol. Conf., May 2008, pp. 61-65.

[11] J. Manssour, I. A. Yafawi, and S. B. Slimane, "Generalized multiplicative network coding for the broadcast phase of bidirectional relaying," in Proc. IEEE Globecom Workshop, Dec. 2011, pp. 1336-1341.

[12] J. Manssour, J. Du, and M. Xiao, "Network-coding-aware link adaptation for wireless broadcast transmission," Telekommunikation, pp. 1-5, Aug. 2013.

[13] W. Chen, Z. Cao, and L. Hanzo, "Maximum Euclidean distance network coded modulation for asymmetric decode-and-forward two-way relaying," IET Commun., vol. 7, no. 10, pp. 988-998, Jul. 2013.

[14] B. Choi and L. Hanzo, "Optimum mode-switching-assisted constantpower single-and multicarrier adaptive modulation," IEEE Trans. Veh. Technol., vol. 52, no. 3, pp. 536-560, May 2003.

[15] J. Torrance and L. Hanzo, "Optimisation of switching levels for adaptive modulation in slow Rayleigh fading," Electron. Lett., vol. 32, no. 13, pp. 1167-1169, Jun. 1996.

[16] A. J. Goldsmith and S. G. Chua, "Variable-rate variable-power MQAM for fading channels," IEEE Trans. Commun., vol. 45, no. 10, pp. 1218-1230, Oct. 1997.

[17] A. J. Goldsmith and S. G. Chua, "Adaptive coded modulation for fading channels," IEEE Trans. Commun., vol. 46, no. 5, pp. 595-602, May 1998.

[18] M. S. Yee, T. H. Liew, and L. Hanzo, "Burst-by-burst adaptive turbocoded radial basis function-assisted decision feedback equalization," IEEE Trans. Commun., vol. 49, no. 11, pp. 1935-1945, Nov. 2001.

[19] T. H. Liew and L. Hanzo, "Space-time trellis and space-time block coding versus adaptive modulation and coding aided OFDM for wideband channels," IEEE Trans. Veh. Technol., vol. 55, no. 1, pp. 173-187, Jan. 2006.

[20] L. Hanzo, C. H. Wong, and M. S. Yee, Adaptive Wireless Transceivers. Turbo-Coded, Turbo-Equalised and Space-Time Coded TDMA, CDMA and OFDM Systems. Hoboken, NJ, USA: Wiley, 2002.

[21] Y. Yang, W. Chen, O. Li, and L. Hanzo, "Variable-rate, variable-power network-coded-QAM/PSK for bi-directional relaying over fading channels," IEEE Trans. Commun., vol. 62, no. 10, pp. 3631-3643, Oct. 2014.

[22] A. J. Aljohani, X. N. Soon, and L. Hanzo, "TTCM-aided rate-adaptive distributed source coding for Rayleigh fading channels," IEEE Trans. Veh. Technol., vol. 63, no. 3, pp. 1126-1134, Mar. 2014.

[23] G. Ungerboeck, "Trellis-coded modulation with redundant signal sets Part II: State of the art," IEEE Commun. Mag., vol. 25, no. 2, pp. 12-21, Feb. 1987.

[24] G. Ungerboeck, "Channel coding with multilevel/phase signals," IEEE Trans. Inf. Theory., vol. IT-28, no. 1, pp. 55-67, Jan. 1982.

[25] Q. Liu, S. Zhou, and G. B. Giannakis, "Cross-layer combining of adaptive modulation and coding with truncated ARQ over wireless links," IEEE Trans. Wireless Commun., vol. 3, no. 5, pp. 1746-1755, Sep. 2004.

[26] A. Goldsmith, Wireless Communications. Cambridge, U.K.: Cambridge Univ. Press, 2005.

[27] G. Ungerboeck, "Trellis-coded modulation with redundant signal sets Par I: Introduction," IEEE Commun. Mag., vol. 25, no. 2, pp. 5-11, Feb. 1987.

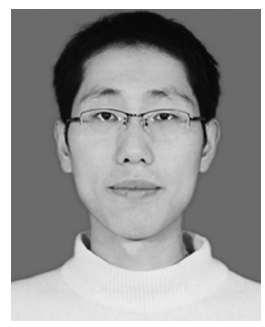

coding.

Mr. Yang currently serves as a Reviewer for the IEEE JOURNAL ON SELECTED AREAS IN COMMUNICATIONS, the IEEE TRANSACTIONS ON VEHICULAR TECHNOLOGY, IEEE COMMUNICATIONS LETTERS, IEEE WIRELESS COMMUNICATIONS LETTERS, etc.

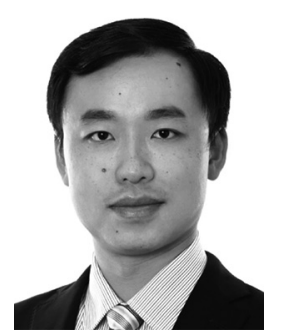

Wei Chen (S'05-M'07-SM'13) received the B.S. degree in operations research and the Ph.D. degree in electronic engineering (both with the highest honors and thesis awards) from Tsinghua University, Beijing, China, in 2002 and 2007, respectively. From 2005 to 2007, he was also a visiting Ph.D. student with the Hong Kong University of Science and Technology, Clear Water Bay, Hong Kong.

Since July 2007, he has been with the Department of Electronic Engineering, Tsinghua University, where he has been a Full Professor since 2012, as a special case of early promotion. After the human resource reform at Tsinghua University, he was elected as a tenured Full Professor of the new research and teaching track in 2015. He also serves as the Deputy Department Head and the University council member and is supported by the National 973 Youth Project, the NSFC excellent young investigator project, the national 10000-talent program, the new century talent program of Ministry of Education, and the Beijing nova program. He visited the University of Southampton, Southampton, U.K. Telecom ParisTech, Paris, France; and Princeton University, Princeton, NJ USA, in 2010, 2014, and 2016, respectively. His research interests include the areas of wireless communications and information theory.

Dr. Chen received the First Prize of the 14th Henry Fok Ying-Tung Young Faculty Award, the Yi-Sheng Mao Beijing Youth Science and Technology Award, the 2010 IEEE Comsoc Asia Pacific Board Best Young Researcher Award, the 2009 IEEE Marconi Prize Paper Award, the 2015 CIE information theory new star award, the Best Paper Awards at IEEE ICC in 2006, IEEE IWCLD in 2007, and IEEE SmartGirdComm in 2012. He holds the honorary titles of Beijing Outstanding Teacher and Beijing Outstanding Young Talent $\mathrm{He}$ is the Champion of the First National Young Faculty Teaching Competition and a winner of National May 1st Medal. He serves as an Editor for the IEEE TRANSACTIONS ON COMMUNICATIONS, the IEEE TRANSACTIONS ON EDUCATION, IEEE WIRELESS COMMUNICATIONS LETTERS, and a Co-Chair of communications theory symposium in IEEE Globecom for 2017. He served as a Tutorial Co-Chair of IEEE International Conference on Communications (ICC) in 2013, a Technical Program Committtee Co-Chair of IEEE Vehicular Technology Conference in the Spring of 2011, and symposium co-chair for IEEE ICC, International Conference on Communications in China, Consumer Communications and Networking Conference, Chinacom, and Wireless and Optical Communications Conference. 
963

962

965

966

967

968

969

970

971

972

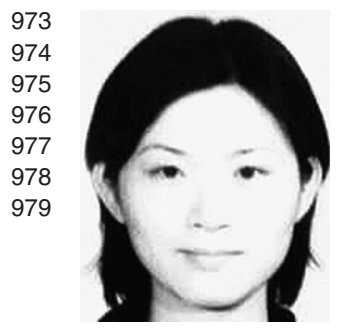

Ou Li received the Ph.D. degree from the National Digital Switching System Engineering and Technological R\&D Center (NDSC), Zhengzhou, China, in 2001

He is currently a Professor with NDSC. His primary research interests include wireless communication technology, wireless sensor networks, cognitive radio networks, multiple input, multiple output, and spectrum sensing.

Ke Ke is currently a Lecturer with the National Digital Switching System Engineering and Technological R\&D Center, Zhengzhou, China. Her research interests include the areas of cognitive communication, wireless ad hoc networks, heterogeneous network convergence, and wireless network security.

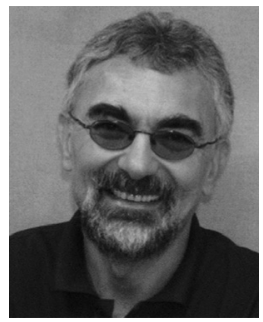

Lajos Hanzo (F'08) received the M.S. degree in 980 electronics and the Ph.D. degree from the Techni- 981 cal University of Budapest, Budapest, Hungary, in 982 1976 and 1983, respectively. He received the presti- 983 gious Doctor of Sciences Research degree in wireless 984 communications from the University of Southamp- 985 ton, Southampton, U.K., in 2004.

In 2016, he joined the Hungarian Academy of Sci- 987 ence, Budapest, Hungary. During his 40-year career 988 in telecommunications, he held various research and 989 academic posts in Hungary, Germany, and the U.K. 990 Since 1986, he has been with the School of Electronics and Computer Science, 991 University of Southampton, U.K., where he holds the Chair in telecommunica- 992 tions. He has successfully supervised $111 \mathrm{Ph} . \mathrm{D}$. students, coauthored $20 \mathrm{John} 993$ Wiley/IEEE Press books on mobile radio communications, totaling in excess of 994 10000 pages, published $1600+$ research contributions on IEEE Xplore, acted 995 both as a Technical Program Committee member and the General Chair of IEEE 996 conferences, presented keynote lectures, and received a number of distinctions. 997 Currently he is directing a 60-strong academic research team, working on a 998 range of research projects in the field of wireless multimedia communications 999 sponsored by industry, The Engineering and Physical Sciences Research Coun- 1000 cil, U.K., and The European Research Council's Advanced Fellow Grant. He 1001 is an enthusiastic supporter of industrial and academic liaison, and he offers 1002 a range of industrial courses. He has $25000+$ citations and an $\mathrm{H}$-index of 60. 1003 For further information on research in progress and associated publications, see 1004 http://www-mobile.ecs.soton.ac.uk.

Dr. Hanzo is a Governor of the IEEE VeHICULAR TECHNOLOGY SocIETY. 1006 During 2008-2012, he was the Editor-in-Chief of the IEEE Press and a Chaired 1007 Professor with Tsinghua University, Beijing, China. In 2009, he received an 1008 honorary doctorate Award by the Technical University of Budapest and in 2015, 1009 from the University of Edinburgh, Edinburgh, U.K., as well as the Royal Soci- 1010 ety's Wolfson Research Merit Award. He is a Fellow of the Royal Academy of 1011 Engineering, The Institution of Engineering and Technology, and EURASIP. 1012 
Q1. Author: Please check the edits made to the sentence "NC methods conceived for multiuser communications were ..." for 1015 correctness.

Q2. Author: Please provide the expansion of "OFDM."

Q3. Author: Please check whether the edits made to the sentence "This is a high-dimensional multivariable discrete optimization 1018 ..." retain the intended sense.

Q4. Author: Please provide volume number for Ref. [12].

Q5. Author: Please provide educational details (degree, subject, institution/university, year) for "K. Ke." 